\title{
Germanica
}

\section{D'Inferno à la Dante-Prosa : Peter Weiss et l'« enfer » ouest-allemand}

Von Inferno bis zur Dante-Prosa. Peter Weiss und die westdeutsche «Hölle»

\section{André Combes}

\section{OpenEdition}

\section{Journals}

Édition électronique

URL : http://journals.openedition.org/germanica/513

DOI : 10.4000/germanica.513

ISSN : 2107-0784

\section{Éditeur}

Université de Lille

\section{Édition imprimée}

Date de publication : 1 juin 2008

Pagination : 47-79

ISBN : 978-2-913857-21-6

ISSN : 0984-2632

\section{Référence électronique}

André Combes, « D'Inferno à la Dante-Prosa : Peter Weiss et l'« enfer » ouest-allemand », Germanica [En ligne], 42 | 2008, mis en ligne le 01 juin 2010, consulté le 06 octobre 2020. URL : http:// journals.openedition.org/germanica/513; DOI : https://doi.org/10.4000/germanica.513

Ce document a été généré automatiquement le 6 octobre 2020.

(C) Tous droits réservés 


\title{
D'Inferno à la Dante-Prosa : Peter Weiss et l'« enfer » ouest-allemand
}

\author{
Von Inferno bis zur Dante-Prosa. Peter Weiss und die westdeutsche «Hölle»
}

\section{André Combes}

Oft genug taucht Dante jetzt wieder am Rande des Auschwitz-Prozesses auf. Man spricht von ,dantesken Szenen'. [...] Auschwitz mit Dantes Inferno zu vergleichen ist fast eine Frechheit,

falls nicht Unwissenheit mildernd ins Feld geführt werden kann. Im Inferno werden schließlich die ,Sünden' von ,Schuldigen' gesühnt.

Dem Inferno folgen immerhin noch Purgatorio und Paradiso. Die Menschen in Auschwitz wären grauenhaft überfragt gewesen, wenn sie einem

durchwandelnden Dante hätten die Sünden aufsagen sollen, um derentwillen sie da gequält wurden. Und ihrer Qual folgte lediglich die Vernichtung. Woher kommt aber die Neigung, die

SS-Chargen für ,Teufel' und ,Bestien' zu halten und die Qualen der Menschen mit Dante zu umschreiben, also aus Auschwitz eine ,Hölle' zu machen? Sicher auch daher, dass für den Berichterstatter Auschwitz einfach keine Realität ist. Wer dem Prozess zusieht, kann ohne weiteres

feststellen, dass Auschwitz nur noch für die „Häftlinge', die überlebten, etwas Wirkliches ist. [...]Weil wir uns also nicht hineindenken können in die Lage der ,Häftlinge', weil das Maß ihres

Leidens über jeden bisherigen Begriff geht und weil wir uns deshalb auch von den unmittelbaren 
Tätern kein menschliches Bild machen können, deshalb heißt Auschwitz eine Hölle und die Täter sind Teufel. So könnte man sich erklären, warum immer, wenn von Auschwitz die Rede ist, solche aus unserer Welt hinausweisenden Wörter gebraucht werden. Nun war aber Auschwitz nicht die Hölle, sondern ein deutsches Konzentrationslager. Und die ,Häftlinge' waren keine Verdammten oder Halbverdammten eines christlichen Kosmos, sondern unschuldige Juden,

Kommunisten und so weiter. Und die Folterer waren keine phantastischen Teufel, sondern Menschen wie du und ich. Deutsche, oder solche, die es werden wollten. [...]. (Martin Walser : Unser Auschwitz)

1 Les années soixante sont pour Peter Weiss des années décisives dans le processus de subjectivation qui l'amènera à effectuer un travail d'anamnèse critique sur l'évolution de son existence et de sa conscience historique et politique depuis les différentes émigrations de la fin des années trente. L'objectif, qu'il poursuivra toute sa vie par-delà les accès dépressifs et la maladie: se constituer une subjectivité affectivement et intellectuellement viable qui lui donne prise sur un monde dont il souhaitait profondément la transformation sans être jamais sûr des formes que celle-ci pourrait prendre. Ceci vaut pour la pensée et l'action politique comme pour la pratique littéraire et artistique : après l'abandon des activités de peintre et de cinéaste dans les années cinquante, ce seront la prose romanesque, la prose autofictionnelle et le texte théâtral qui formeront le vaste intertexte où se déploiera ce processus de subjectivation.

2 Ce fut un «Selbstverständigungsprozess » infini dont parle fréquemment la recherche weissienne qui en repère les figurations complexes depuis les textes largement autofictionnels du début des années soixante, Abschied von den Eltern (1961) et Fluchtpunkt (1962) jusqu'à Ästhetik des Widerstands, le grand œuvre de la dernière décennie d'existence de Peter Weiss : après l'intense période de production théâtrale des années 1963-1972 ${ }^{1}$, il marque un retour à la prose romanesque comme autre forme de théâtralité.

3 Ce processus semble avoir trouvé dans les années soixante ses scansions essentielles autour de trois axes centraux placés sous le signe de l'appropriation ou de la réappropriation : judéité (à faire advenir), langage poétique (à redécouvrir dans la langue maternelle) et prise de parti politique inscrite «dans un monde divisé $»^{2}$. Ils forment l'armature du travail obstiné et souvent douloureux sur la manière dont l'histoire individuelle de Weiss s'est nouée à l'histoire collective, surtout celle des années trente et quarante qu'il pensait avoir profondément méconnue ou mal comprise, occupé qu'il était à arpenter les très inconfortables " prisons de l'intérieur » dont parle Sade dans le Marat/Sade, le grand texte dramatique de 1963 qui lui valut une audience internationale.

4 Weiss accomplit ainsi dans ces années soixante un travail de prospection de sa biographie et de sa mémoire des événements qui la tissaient et/ou l'entouraient tout en vivant une "rémigration » intellectuelle difficile dans un État ouest-allemand qu'il ressent comme profondément « unheimlich » même s'il y est publié et s'il y noue ses 
principaux contacts (Groupe 47 et Suhrkamp Verlag). Ce retour dans des territoires qu'il avait quittés avec sa famille en 1935 pour l'Angleterre, avant les séjours à Prague et en Suisse puis l'émigration définitive en Suède en 1939 - il deviendra citoyen suédois en 1946 et écrira ses premiers textes en suédois - s'articula entre 1964 et 1969 sur un immense projet littéraire, le " projet Divine Comédie " ${ }^{3}$ qui nourrira également le travail sur une poétique engagée de l'histoire entrepris dans Die Ästhetik des Widerstands ${ }^{4}$. Notons rapidement ici que la recherche weissienne a, elle aussi, posé le problème de la légitimité d'un recours métaphorique à l'Enfer de Dante, qu'un Martin Walser avait fortement critiqué dès 1965 dans Unser Auschwitz, pour décrire la réalité de l'expérience des camps de la mort et surtout pour être au plus près d'une vérité subjective de la représentation de celle-ci, comme si puiser dans un fond apparemment connu voire familier (mais souvent très méconnu) d'images littéraires de la terreur s'imposait paradoxalement pour promouvoir une parole authentique ${ }^{5}$.

Le projet est donc de réécrire la trilogie dantesque, Inferno/Purgatorio/Paradiso. Il demeurera largement inachevé mais l'ouvrage fut quinze années durant régulièrement remis sur le métier: les textes successifs - œuvres publiées (Paradiso/Die Ermittlung), œuvres restées longtemps inédites (Inferno) ou témoins de genèse - en sont aujourd'hui bien connus ${ }^{6}$. De la trilogie weissienne, Inferno, écrit dans la deuxième moitié de l'année 1964, un an après le Marat/Sade, devait être le premier volet. Face aux hésitations de Siegfried Unseld, le « patron » du Suhrkamp Verlag, et de son «Cheflektor » Karl-Heinz Braun à publier la pièce qui restera inédite jusqu'en 2003, Weiss n'abandonne pas pour autant un projet qui connait sa phase de travail la plus intense et la plus productive dans les années 1964-1969. À l'été 1965, il termine la réécriture du troisième volet de la trilogie, Paradiso, immédiatement publié par Suhrkamp sous le titre de Die Ermittlung ${ }^{7}$ avant d'être représenté pour la première fois en octobre de la même année sur une quinzaine de scènes différentes de RFA et de RDA ${ }^{8}$. Purgatorio restera, quant à lui, à l'état d'ébauche. Dans les années 1965-1969, ce sont surtout les textes dramatiques déjà mentionnés qui portent trace du travail effectué sur la Divine Comédie avant la clôture textuelle effective du projet que représente la Dante-Prosa en 1969.

6 Ce projet de tramer un "Welttheater » contemporain sur le «Weltgedicht » que fut à ses yeux l'épopée de Dante révèle d'abord une volonté d'interpeller un grand texte pour revisiter après coup et de manière indissociable sa propre biographie et l'histoire allemande des dernières décennies: on peut y voir la volonté de comprendre son propre vécu par une représentation qui ne soit pas pure introspection ${ }^{9}$ et de redéfinir, d'un même mouvement, son propre rapport à la littérature et à l'art. La réécriture de Dante est ainsi au fondement de la re-mémoration / perlaboration contradictoire des événements passés pour faire sauter les verrous du refoulement et de l'occultation qui barraient le chemin de la connaissance : celui que le Je-narrateur de la Divine Comédie, vigoureusement soutenu par Virgile, emprunte au début de L'Enfer et que le Dante-» Heimkehrer » fictionnel d'Inferno ressentira comme une prise de conscience balbutiante et assimilera aux instants qui précédent la mort, quand «le monde est encore là mais plus la connaissance $»^{10}$.

7 Au centre biographique et historique du " projet Divine Comédie », Weiss placera dans un premier temps le nazisme et la Shoah, dont il percevra les effets pérennes dans la RFA des années soixante et auquel il mêlera ses propres refoulements. Le sentiment tenace de son autisme idéologique et politique dans l'émigration suédoise - qui ne fut pas exil au sens du poème de Brecht « Über die Bezeichnung Emigranten » $(1937)^{11}$ - va 
ainsi contribuer à faire émerger après coup un puissant « syndrome du survivant » qui sera lié à celui de ne pas avoir accompli, à l'inverse de son Dante fictionnel, un véritable retour d'exil : Inferno mettra par ailleurs en scène, avec une douloureuse insistance, le très fort sentiment de culpabilité envers les victimes de la Shoah - nourri d'une profonde incertitude rétrospective sur un engagement possible aux côtés des bourreaux - qui contraint Peter Weiss à réinterroger compulsivement sa biographie par le truchement d'un Dante à la fois historique et fictionnel. Les différentes versions du texte montreront comment l'écriture le constitue obstinément en une sorte de centre condensé et déplacé d'un traumatisme fantasmé qui pourra par exemple se cristalliser autour de la métamorphose de la Béatrice dantesque en "Bea", double fictionnel vraisemblable de l'amie de jeunesse Lucie Weinberger ${ }^{12}$. L'engagement politique d'après 1965 pourra alors se lire comme "Freispruch von der eigenen Verschuldung ${ }^{13}$. Sur ce point essentiel, le Dante d'Inferno ou celui, devenu jenarrateur, de la Dante-Prosa - les deux textes auxquels je m'attacherai dans ce travail se démarqueront irréductiblement du Dante/je-narrateur de la Divine Comédie. Dans Die Ästhetik des Widerstands, l'un des personnages tramé sur un résistant allemand réel, Hans Coppi, notera rétrospectivement qu'aucun sentiment de culpabilité n'effleurait la bonne conscience chrétienne du Dante qui s'autofictionnalisa dans La Divine Comédie :

Doch bleibt dieser Moralist [...], der jeden seiner Feinde und Antagonisten der Verdammung ausliefert, selbst unbeschmutzt, er kann wohl weinen, kann in Ohnmacht sinken, angesichts all dieser Schmerzen [...], nie aber trifft ihn der Gedanke, dass auch er durch ein Zögern, eine Unterlassung, ein Verschweigen, ein Leugnen in den Augen eines andern schuldig wird, er geht durch das Böse hindurch und weiß, solange er sich an der Hand des Schutzgeists, des künstlerischen Bewusstseins (représenté par Virgile A. C.), festhält, kann ihn nichts behelligen ${ }^{14}$.

Pour comprendre le projet de s'attaquer à la réécriture de Dante, il faut également mentionner l'importance des procès d'Auschwitz de Francfort sur le Main (décembre 1963 à août 1965) ${ }^{15}$, qui jouèrent un rôle capital dans le déclenchement du processus subjectif de confrontation à la Shoah, médiatisée par la lecture du poète florentin. En décembre 1964 - il venait alors de terminer la rédaction d'Inferno - Weiss accompagna la délégation du tribunal francfortois qui se rendit à Auschwitz. Comme il l'écrivit à Hans-Werner Richter, il avait besoin d' 'impressions directes d'Auschwitz " pour achever le troisième volet de sa trilogie (Paradiso/Die Ermittlung) ${ }^{16}$. Le texte qu'il rédigea au retour de cette visite et qu'il intitula significativement " Meine Ortschaft ", décrit - avec le sentiment que l'accumulation des connaissances quantitatives, statistiques sur Auschwitz est à la fois nécessaire et définitivement insuffisante l'appropriation rendue possible après coup d'un lieu vidé de ses horreurs mais, pour celui qui sait se mettre à leur écoute, encore pleins du bruissement des fantômes. Ceuxci ne demandent qu'à faire retour dans le présent et invitent Weiss à entrer - à l'instar d'un Dante contemporain - dans le royaume des morts. La rencontre avec cette «localité » qui aurait pu être sa dernière destination si l'émigration ne l'en avait préservé, comme le rappellera Aischmann-Dante dans la version 8 d'Inferno ${ }^{17}$, et qui n'était pas encore devenu un « lieu de mémoire ", aura une importance capitale pour le travail réflexif intense sur les significations à la fois individuelles et collectives d'une Shoah découverte si tardivement et à laquelle il ne pouvait s'identifier qu'en soulignant de fondamentales différences :

Ich bin hierher gekommen aus freiem Willen. Ich bin aus keinem Zug geladen worden. Ich bin nicht mit Knüppeln in dieses Gelände getrieben worden. Ich komme zwanzig Jahre zu spät hierher ${ }^{18}$. 
9 On peut poser, dans ce contexte, plusieurs questions dont les plus importantes pourraient se formuler ainsi :

10 - A-t-il fallu la rencontre après coup de la biographie de Peter Weiss et du " grand récit " de la Shoah qui émerge dans les années soixante, quand, dans le sillage des procès d'Auschwitz de Francfort sur le Main (décembre 1963 à août 1965), le " paradigme Auschwitz » remplace le " paradigme Buchenwald », pour lui permettre de donner une inflexion différente à sa subjectivation en y intégrant par exemple une composante juive longtemps méconnue ${ }^{19}$ ?

11 - Ces procès ont-ils inspiré le désir de scruter dans la Divine Comédie l'opiniâtreté d'un véritable poète qui ne se laisse pas décourager par les vacillements de la langue poétique devant les visions infernales que Weiss constatait pour lui-même lorsqu'il écrivait :

Die Möglichkeit des Sprechens / ging mir schon verloren, als ich mich bemühte, die Eindrücke / dieses Tribunals festzuhalten und mir die Geschehnisse vorzustellen, / die der Verhandlung zugrund lagen. Meine Gedanken versagten, als ich / an die Reichweite dachte dessen, das hier angerührt wurde. / Trotz zahlreicher Anfälle von Mutlosigkeit und Überdruss gelang es Dante / Worte zu finden für einen Stoff, der allen gehörte, doch ungreifbar schien, / und was sich bisher der Sprache entzogen hatte, war jetzt / vernehmbar. Gesichten gab er Form, die in den Träumen seiner Zeitgenossen / lebten, und Kunst war ein Mittel, Gesichte zur Sprache kommen zu lassen $?^{20}$.

12 - Y avait-il chez Weiss, le sentiment culpabilisant d'être passé à côté de l'Histoire et de son histoire ${ }^{21}$, celle du nazisme, de l'exil mais aussi celle de la résistance antifasciste, en particulier celle du mouvement ouvrier, redécouvertes véritablement - après quelques confrontations cinématographiques de l'immédiat après-guerre ${ }^{22}$ - lors du travail sur le « projet Divine Comédie » et sur Die Ästhetik des Widerstands?

13 - Cela l'amènera-t-il à réécrire fantasmatiquement son existence dans Die Ästhetik des Widerstands, "Wunschautobiographie » dans laquelle un je-narrateur questionne la légitimité de l'existence du poète et de l'artiste à l'ère de l'oppression généralisée, ce en quoi Dante avait pu fournir un modèle de réécriture : poète engagé dans les clivages politiques florentins de son temps, obligé de quitter Florence pour Vérone en abandonnant les siens, plusieurs fois condamné à mort par contumace, puis se séparant de ses compagnons d'exil pour mourir à Ravenne sans avoir revu Florence?

14 - Y avait-il enfin le sentiment que la culture dominante - y compris celle de l'aprèsnazisme - refusait à l'écrivain, comme il le dira lors d'une interview de l'automne 1975 sur Die Ästhetik des Widerstands ${ }^{23}$, l'accès aux valeurs culturelles véritables qui lui sont nécessaires pour exprimer les problèmes philosophiques ou esthétiques de son temps, offrant au "rémigrant » un soutien ressenti comme profondément ambigu qui masque à peine une hostilité à la fois bienveillante et arrogante, comme le décriront longuement les Chants 1 et 3 de la Dante-Prosa?

15 Alors que Weiss, lors de la rédaction d'Inferno en 1964, n'avait vraiment lu que le premier volet de la trilogie de Dante, L'Enfer,il a entre 1964 et 1969 approfondi l'œuvre comme le manifestera la Dante-Prosa qui arpente plus nettement certains cercles de L'Enfer dantesque. Par ailleurs, les deux textes déjà mentionnés - la Vorübung zum dreiteiligen Drama Divina Commedia, texte en vers libres rédigé parallèlement au travail sur Inferno, et le Gespräch mit Dante, dialogue fictif qui est une sorte d'interview de " A » - Peter Weiss sur son projet et qui fut écrit au printemps 1965 peu après le 
remaniement de Paradiso/Die Ermittlung ${ }^{24}$ - amorcent, au même moment, une réflexion approfondie sur les enjeux personnels, historiques et esthétiques du "projet Divine Comédie ». Ces deux textes que l'on lie souvent ensemble révèlent une évolution notable du projet, le second proposant une extension de la représentation de la terreur à des événements postérieurs au nazisme et à la Shoah : «Aus der Erinnerungsarbeit für die Ermordeten des deutschen Faschismus erwächst eine Solidarisierung mit sämtlichen Verfolgten, die weltweit unter politischen oder materiellen Zwängen leiden », écrit Yannick Müllender ${ }^{25}$ qui voit dans cette " évolution d'un "drame allemand" » centré sur la division entre «Täter » et "Opfer », à un « théâtre du monde universel » mettant en scène "Unterdrücker » et "Unterdrückte », la modification principale apportée au projet à partir du deuxième semestre 1965, celui où Weiss va passer sur des positions ouvertement anticapitalistes et antiimpérialistes avec la publication des 10 Arbeitspunkte eines Autors in der geteilten Welt. Auschwitz devient alors le « chiffre » d'un "principe de la persécution et de l'exploitation de vastes populations jusqu'à l'extermination » inhérent "à la structure des états capitalistes ${ }^{26}$. Alors que dans les différentes versions d'Inferno Weiss mettra en scène un double fictionnel de lui-même qui reconnaîtra que l'exil l'avait préservé de développer des tendances qui auraient pu faire de lui un bourreau ${ }^{27}$, il fera de ce topos dans Die Ermittlung une possibilité générale inscrite dans les structures sociales, comme l'énonce le « témoin 3 » au Chant 3 :

Viele von denen die dazu bestimmt wurden / Häftlinge darzustellen / waren aufgewachsen unter den selben Begriffen / wie diejenigen / die in die Rolle der Bewacher gerieten /.../ und wären sie nicht zum Häftling ernannt worden / hätten auch sie einen Bewacher abgeben können.

Néanmoins, comme le dira le même témoin, c'est bien avec Auschwitz que ce principe connaîtra son "ultime conséquence", le point de non retour où se brise toute civilisation. Le « témoin 3 » rejoint ici l'opinion de Peter Weiss :

Die Ordnung die hier galt / war uns in ihrer Anlage vertraut / deshalb konnten wir uns auch noch zurechtfinden / in ihrer letzten Konsequenz / in der der Ausbeutende in bisher unbekanntem Grad / seine Herrschaft entwickeln durfte / und der Ausgebeutete / noch sein eigenes Knochenmehl liefern musste ${ }^{28}$.

Mais, à mon sens, l'extension thématique qui prend l'aspect d'une relativisation voire d'une historicisation d'Auschwitz - «Todesfabrik, die unter bestimmten Umständen überall existieren könnte $»^{29}$ - n'est pas pour autant une relativisation des rôles historiques des bourreaux et des victimes, si on les réfère au phénomène incommensurable que fut Auschwitz dont on peut penser que sa singularité le rendit précisément en ce sens universel qu'il fallait tout faire pour en éviter le retour : comme répétition ou comme extension. Weiss tirait, pour la représentation esthétique, les conséquences des préceptes philosophiques et politiques d'Adorno: "...Denken und Handeln so einzurichten, dass Auschwitz sich nicht wiederhole, nichts Ähnliches geschehe ${ }^{30}$. Dans la version d'Inferno la plus soucieuse de nourrir l'action présente des enseignements de la mémoire (Aischmann-Fassung), Dante-Aischmann revient sur l'aveuglement passé devant les signes insistants de la catastrophe et sur leur évidence rétrospective qui est une injonction envers le présent à ne pas laisser l'aveuglement se reproduire alors que les signes se sont aujourd'hui « accrus » :

Wenn wir uns heute mit dieser Zeit befassen / so sehen wir deutlich die Zusammenballung / die zu einer Entladung führen musste /.../ Die Zeichen vor denen wir uns damals verschlossen / sind noch vorhanden / sie haben sich erweitert sind schwerer zu greifen / überlagern uns aber nach wie vor (Mü, 440). 

versions conservée et transformée une constellation restreinte de personnages de la trilogie dantesque, comme nous le verrons, alors que la version 0 du plan général détaillé en 33 Chants en mettait en scène un très grand nombre en suivant en grande partie leur répartition dans les différents cercle de L'Enfer de Dante. Sera également conservée l'architecture ternaire Inferno / Purgatorio / Paradiso. Mais celle-ci sera sécularisée et réinterprétée pour pouvoir dire non plus le monde terrestre du XIII siècle, allégorisé du point de vue de la transcendance chrétienne et croisé avec les topographies de l'univers imaginées par Ptolémée ${ }^{32}$, mais celui des continuités et restaurations allemandes des deux premières décennies de l'après-guerre telles que Peter Weiss les découvrit au début des années soixante sur fond des révélations apportées par les procès d'Auschwitz ${ }^{33}$. La prise de conscience de ce que signifièrent ces procès - autant pour la compréhension des réalités des camps d'extermination que des réalités ouest-allemandes - imposa une inversion fondamentale de la «Jenseitsarchitektur» (Peter Kuon) de l'œuvre de Dante afin d'en manifester l'inactualité fondamentale " après Auschwitz » :

Der erste Teil ist das Inferno, der zweite das Purgatorio, der dritte das Paradiso ${ }^{34}$. Es treten auch die gleichen Figuren wie in Dantes Epos auf - natürlich in unsere moderne Zeit versetzt. [...] Im Inferno werden nach Dantes Vorstellung die Sünder mit ewigen Höllenqualen bestraft. Aber heute werden sie nicht bestraft und sind nicht in der Hölle. Sie leben in dieser Welt weiter mit ihrer Schuld und werden niemals bestraft. Diese Menschen, die an sich bestraft werden müssten, leben sehr bequem unter uns als Herrschende und Machthaber. Und die im Paradies leben sollten, leben ewig in dem Elend, das sie durchgemacht haben. [...] In diesem Paradies-Teil (= Die Ermittlung, A.C) ist eine Menge Material über die Konzentrationslager verwendet. Ich habe den Auschwitz-Prozeß oft besucht und dadurch viel Material bekommen. [...] Das Mittelstück, das Purgatorio, soll genau den Zustand des Zweifels ausdrücken. Es ist die Welt, in der wir leben, wo wir nicht genau wissen, wie wir uns entscheiden sollen, auf welcher Seite wir stehen sollen diese absolut unentschiedene Situation ${ }^{35}$.

On peut également citer ici ce passage du Gespräch über Dante sur les tâches qui attendraient un Dante contemporain :

So wie er im Inferno-Teil die Mächtigen dieser Welt in ihren Hochburgen darstellt und dabei deutlich macht, dass ihre Herrschaft noch ungebrochen ist, führt er uns im Paradiso die Seligen vor, die immer noch auf ihre Befreiung warten ${ }^{36}$.

Le paradis des puissants sera donc l'enfer du Dante de Peter Weiss dans Inferno. Un passage des Notizbücher 1960-71 actualise même sans équivoque cet enfer : il est devenu «Die Welt der Großgangster ». Le plan général de la version 0 y pointera l'omniprésence du complexe militaro-industriel qu'évoqueront quelques passages de Die Ermittlung et qui sous-tend l'extension thématique de la Shoah (voir supra) :

Die internationale Klique der Grossindustriellen die Rüstungsdirektoren Chefs der chemischen Fabrique die vor dem Krieg während des Krieges und nach dem Krieg einander in die Hände spielten die Aktien die Aufsichtsräte die Kriegsgewinnler =Massenmörder (Mü, p. 342).

21 Dans un entretien avec Hans Mayer sur Die Ermittlung d'octobre $1965^{37}$, Weiss précise les implications plus générales et plus directement politiques de cette "perspective inversée » qui ne permet pas seulement une lecture actuelle de la RFA des années soixante, devenue le " paradis » des anciens bourreaux et de l'« enfer » pérennisé des victimes, mais pose la question plus générale du statut idéologique de l'enfer et du 
paradis chrétiens, revisités mais non abolis par Dante, pour le destin terrestre des humains. Reprenant la logique de la lutte pour le socialisme dont il se réclamait depuis septembre 1965, Weiss souligne que c'est de leur vivant que les humains doivent lutter pour faire cesser leurs souffrances :

Die Ausgangsüberlegung war, dass nach des alten Dante Ansicht die Menschen für ihre Leiden nach dem Tod mit dem ewigen Paradies belohnt werden sollten, während in unserer Welt diese Möglichkeit nicht mehr besteht. Die Menschen, die mit ihren Leiden sterben, haben keine Belohnung zu erwarten und deshalb nur die einzige Möglichkeit, sich zeit ihres Lebens von den Leiden zu befreien und nicht erst nach dem Tode.

Se dessine ici une extension de la problématique de l'«inversion» des signifiés de la trilogie de Dante qui prolonge et excède la Shoah: si les bourreaux avaient réussi à transformer en paradis ce qui aurait dû devenir en bonne justice leur enfer, il faut hic et nunc créer les conditions d'un retournement de cet inique détournement :

Die einzige Möglichkeit, sie an ihren Taten zu hindern, wäre, sie hier - also auch zeit ihres Lebens - zu bekämpfen. Die Hölle wäre also der Ort der Tyrannen und das Paradies der Ort der Leidtragenden. Auschwitz wäre ein Teil dieser Welt gewesen, jedoch hat sich während der Arbeit dieser Komplex so vergrößert, dass ein selbständiges Stück daraus geworden ist ${ }^{38}$.

Quant à la constellation des personnages de la Divine comédie, elle va subir elle aussi des transformations signifiantes en accord avec le renversement de la topographie de base. C'est sur le personnage principal de la Divine Comédie et son accompagnateur (Dante et Virgile) que va s'opérer la première grande modification, rendue possible par le découplage du je biographique et du je fictionnel, par rapport à l'hypotexte dantesque. Elle fait du Dante d'Inferno un double plus distancié de l'auteur, propre à servir de surface de projection et de réflexion aux éléments à ses yeux les plus problématiques de sa propre biographie tandis que dans la Dante-Prosa, qui marque un repli subjectif de Weiss, celui-ci reprendra le couplage initial de la Divine Comédie entre un je-narrateur et un je-narré, un je-sujet qui se prend lui-même et les événements qu'il vit comme objet de sa narration.

Le premier chant de la version achevée d'Inferno s'ouvre sur une longue didascalie qui décrit l'apparition d'un être "petit et malingre blanc comme craie / dans un vêtement bien trop large et flottant " mais la tête ceinte d'une couronne de laurier, sur le podium d'une baraque foraine où le "chef-patron» («Chef») présente des spectacles improvisés. Celui-ci, en frac, haut de forme et bottes et maniant le fouet, est entouré de 7 personnages dont trois portant le masque d'un lynx, d'un lion et d'une louve, ces animaux symboliques qui horrifiaient le je-narrateur dans le premier Chant de la Divine Comédie, avant que Virgile ne l'encourage à poursuivre sa route, et qui harcèlent ce personnage totalement décati ( auf den Hund gekommen»), que personne ne semble plus connaître, sauf Virgile, apparemment la seule mémoire culturelle de la cité, qui rappelle que Dante fut jadis « condamné à (y) être brûlé ».

Voué au bûcher par ses adversaires et donc contraint à l'exil, comme le Dante réel, le Dante fictionnel de cette ultime version d'Inferno revient dans la ville de ses anciens persécuteurs qui entendent bien le réintégrer totalement, ou plutôt totalitairement, en faisant de lui un vassal intellectuel et moral de l'ordre nouveau reconstruit sur l'ancien : exigence réitérée de Chant en Chant de faire allégeance absolue au nouveau pouvoir et à sa vision du réel, à sa conception mercantile et sans substance de l'art, mais surtout se laisser dicter les modalités d'une mémoire qui, le présentant lui-même 
comme ex-persécuteur et donc co-coupable ${ }^{39}$, va instrumentaliser le «syndrome du survivant " jusqu'à ce point ultime où survivre ne pourra plus signifier que pactiser avec les nouveaux/anciens bourreaux « restaurés » dans leur pouvoir. Le retournement opéré par Weiss sur la «Jenseitsarchitektur » est énoncé sans ambages dès le deuxième Chant d'Inferno : Virgile, sous le regard du " chef-patron » et avant que ne commence le spectacle théâtral que celui-ci va mettre en scène, signifiera au chœur des anciens bourreaux, dans une rhétorique qui mêle pédagogie appuyée et démagogie, cette cardinale «transvaluation »:

Wer glaubt, dass er für seine Sünden in die Hölle käme / der irrt und sei nur frohen Mutes / denn nach dem Tod ist keiner der ihm etwas nähme / Wer Schlechtes tut erwirbt für sich nur Gutes.

Puis il fait entonner par le chœur - "Singt alle mit» - le chant de l'arrogance des bourreaux. «Ein schönes Lied », remarque le « chef-patron » qui en conçoit l'idée d'une pièce dans le style improvisé de la «Stegreifbühne $»^{40}$. Il y a ici, à la différence des différentes versions du texte, une mise en abyme d'un théâtre affirmatif qui apparaît d'entrée comme le théâtre d'un pouvoir qui peut se permettre de re-présenter la tragédie historique des guerres civiles et des persécutions sous forme de farce - ce qui est farce pour le pouvoir étant tragédie pour ses victimes -, manifestant par là-même qu'il n'y a de pouvoir politique absolu, comme l'avaient compris Benjamin et Brecht aux temps du nazisme, que s'il y a aussi pouvoir absolu sur la/les représentation(s).

La domination de celui-ci est ainsi thématisée dès le quatrième Chant d'Inferno qui s'ouvre par une modification radicale de la formule célèbre de Dante à l'entrée de la cité dolente au début du Chant 3 de la Divine Comédie: le "Vous qui entrez ici, abandonnez toute espérance ", devient chez Weiss : "Ihr die ihr kommt / lasst alle Zweifel fahren", la cité étant devenue une ville "dans laquelle il n'y a plus de douleurs $»^{41}$. Ville anesthésiée qui a assis durablement le pouvoir de ceux pour qui oublier, c'est d'abord refouler, réécrire l'histoire du point de vue des puissants, ceux-ci régnant indistinctement et sans partage sur les institutions politiques, économiques et culturelles dont ils prescrivent, « avec le consentement actif des dominés » (Gramsci) ${ }^{42}$, les valeurs et les comportements dominants autant que les contenus de la mémoire: cette mémoire, systématiquement éradiquée de la nouvelle société, étant travestie en « ouverture » et en « liberté » dont pourrait jouir le poète-revenant. Virgile l'énonce au Chant 5 : « Sieh doch wie offen es hier ist / nichts bindet dich / keine Vergangenheit ist dir im Weg / und alles was du willst / das steht dir frei zu tun/». Dans ce chant, Dante est donc, en bonne logique, symboliquement dépouillé de ses attributs des anciens temps - la couronne de laurier, le bâton et le manteau - pour mettre en scène spectaculairement le rite de passage cruel de cette métamorphose : à moitié nu mais portant encore le bonnet immortalisé par le portrait de Giotto, le visage ayant récupéré ses couleurs, l'apparence "désuète » mais "sportive", selon les didascalies, il serait, proclame le « chœur final » du Chant, « lui-même pour la première fois ». Lui qui fut au début de la pièce exposé comme une bête de cirque, il est alors prêt à passer l'« examen d'entrée " exigé pour sa réintégration dans la vie publique et culturelle de sa ville natale. Au Chant 7, un jugement prononcé par le « chef-patron » devenu Minos indique le sens profond de la métamorphose souhaitée :

Aus dieser Eintrittsprüfung geht hervor / dass der der Dante heißt / und uns mit Lorbeer und klassischem Gewand / zu täuschen suchte / um nichts besser ist als wir / Er steckt voll Schummelei und ist ein feiger Hund / ist gierig geizig und auf seinen 
Vorteil bedacht / und jedes Wort das er von Liebe spricht / ist Quatsch / Aus diesem Grund ist er hier aufgenommen / und soll gleich eingekleidet werden. drame à stations qui, structurellement, pourrait illustrer le paradoxe découlant de l'inversion du sens des topographies transcendantales de la Divine Comédie: la montée au paradis des soumis comme descente aux enfers du rémigrant dissident. Dans le «canto 4 » de la version 8, Charon déclarait Dante-Aischmann irrécupérable en calquant au plus près la situation de Peter Weiss au milieu des années soixante. Lui reprochant sa rigidité intellectuelle, il définit celle-ci comme un «halbgegorenes Weltverbessertum, eine völlig verlegene Enttäuschung an einem Sozialismus, der nie lebensfähig war, sowie ein Festhalten an einem verlorenen, wirklichkeitsfernen dritten Standpunkt. » (Mü, 421). Contrairement au personnage de la version achevée d'Inferno, Dante-Aischmann était montré, dès la première réplique de cette même version, métamorphosé en "Heimkehrer» lucide et pugnace, confronté aux survivances tenaces de l'antisémitisme :

Jetzt bin ich wieder da, nach dreissig Jahren. Und was ich hörte, auf dem Weg vom Hotel, an der Autobushaltestelle, das war ein Ausspruch, der die Zeit von damals wachrief. Da hörte ich einen Mann zu seinem Kind sagen: du bist ja wie ein Judenbengel. Ich blieb stehn. [...] Ich sah die Umstehenden. Sie hatten es alle gehört. Niemand kümmerte sich darum. Einer lachte nur. Ich dachte, noch vor zwanzig Jahren hättet ihr mich durch den Kamin gejagt. (Mü, 407).

Mais ce premier chant reprend également certains des thèmes récurrents qui habitent la représentation de la Shoah dans plusieurs Chants des autres versions d'Inferno: le lâche refus d'intervenir qui rappelle des lâchetés analogues dans le passé, motivées par la crainte d'avoir à choisir entre une "fuite folle et aveugle » et la "plus extrême humiliation » (Mü, 408), la mécompréhension du «mal » et le sentiment diffus de la menace pesant sur le « demi-juif » : Aischmann ou Peter Weiss. On voit par ailleurs dans le Aischmann-Entwurf comment l'anticommunisme triomphant dans la RFA des années soixante est inscrit dans la succession idéologique de l'antisémitisme nazi, comme l'explique Charon à Dante-Aischmann :

Heute geht es nicht mehr um die Auseinandersetzung mit kleineren Gruppen, die als Fremdkörper im Volk empfunden werden [...], sondern um die Gegenüberstellung zweier Lebensprinzipien (Mü, 420).

Du personnage principal de l'ensemble des textes qui constituent le "projet-Divine Comédie ", on peut encore noter que Weiss envisagea initialement de le remplacer par Giotto $^{43}$. Mais celui-ci va subir au cours des différentes versions d'Inferno une dévalorisation constante qui le métamorphosera dans la version 10 («Giotto-Entwurf ») - le remaniement le plus important que Peter Weiss lira en septembre 1964 à la rencontre du Groupe 47 à Sigtuna et qui est dramaturgiquement et surtout textuellement le plus proche de la version achevée - en une sorte de substitut de Virgile $^{44}$. Ce dernier ne sera alors plus le "guide, maitre et auteur ", décrit par le jenarrateur dans le premier Chant de L'Enfer et encore envisagé dans la Vorübung ou la conception initiale d'Inferno $0^{45}$, mais une sorte de délégué aus affaires culturelles parfaitement inféodé au nouveau pouvoir et chargé, pour la plus grande gloire de celuici, de « récupérer » l'exilé encore nimbé de sa gloire passée.

31 Les différentes versions du texte montrent le statut narratif et idéologique éminemment changeant de Virgile ${ }^{46}$. Il peut ainsi être potentiellement une instance du moi de Dante, une sorte de moteur interne du cheminement vers la mémoire, la 
conscience et la langue perdues, qui au début d'Inferno était à la fois le seul capable d'identifier le "Heimkehrer " hébété et muet, figurant en quelque sorte la mémoire culturelle d'une cité qui l'avait éradiquée au profit de l'autocélébration du présent ${ }^{47}$. Mais Virgile est fondamentalement, comme le confirme la version achevée d'Inferno, l'homme-lige des différentes hypostases du «Chef/Patron », Minos et surtout Pluto, le ploutocrate-mécène omnipotent que l'on trouve au Chant 9 d'Inferno et que reprend le «Inferno 7 » du «Giotto-Entwurf » (Mü, p. 457 et sq.) en l'accompagnant du «chœur des ploutocrates" et en le coiffant d'un casque qui, avec le frac, le transforme en allégorie syncrétique du capitalisme impérialiste et militariste. On retrouve ce même Pluto dans une grande tirade de " président du conseil d'administration » au « canto 7 » du «Aischmann-Entwurf» qui décrit la «ruche» bourdonnante de modernité matérielle qu'est la nouvelle organisation sociale où cohabitent banques, commerces, universités, théâtres et musées. Cette organisation complexe et sophistiquée, déclare un Pluto lyrique à Dante-Aischmann, est comparable à l'élaboration des "images poétiques » en système versifié (Mü, p. 432). C'est dans cette tirade que Peter Weiss pousse dans ses extrêmes conséquences sa vision de l'intrication du système sociopolitique dominant et d'une pratique poétique qui lui serait structurellement apparentée. Virgile est chargé par cette ploutocratie-là de tenir aux anciens dissidents comme Dante le même discours opportuniste et affirmatif de l'allégeance nécessaire au nouveau monde totalitaire des puissants et à leurs valeurs restaurées afin de les rendre récupérables par un système qui, en prétendant assurer à tous une liberté totale, parachève sa domination sans partage sur l'économie, la société et la culture. Dans la dernière version d'Inferno, c'est au Chant 26 que Virgile énonce sans ambages la perception weissienne de la restauration ouest-allemande en prononçant un éloge appuyé du «Generaldirektor Futschi » :

Merke Alighieri / der große Mäzen der damals Giotto unterstützte / beherrscht auch heut noch die Finanzen / Nicht nur als Vorsitzenden der Aufsichtsräte / unsrer einflussreichsten Industrien / und als Ehrenpräsidenten unseres Verteidigungswesens / sondern auch als unsern größten Förderer / der jungen Generation von Künstlern / verehren wir / Generaldirektor Futschi (p. 92).

Dans la Dante-Prosa, Virgile se transformera en un accompagnateur incertain, à l'identité et à la présence vacillantes voire hypothétiques - «Vergil, wenn es Vergil war » (Mü, 523) - qui n'est ni soutien actif du poète florentin ni non plus vraiment un chargé de mission culturel du pouvoir ${ }^{48}$ : «jemand, dessen Name mir immer wieder entfiel, und den ich Vergil nennen wollte, was ich verwarf, da es einen Vergil für mich nicht geben konnte " (Mü, 507). Il est intéressant d'analyser ici comment il devient même dans certaines versions du premier Chant d'Inferno une sorte de double de Dante, qui dit «je » à sa place mais ne fusionne pas toujours complètement avec lui. Cette fusion partielle subit quelques variations dans les versions 1,2 et 3 du texte. Dans un premier temps, Weiss avait envisagé de faire de Virgile celui qui questionne Dante sur son identité et son origine avant de permuter les rôles et de faire de lui une instance mémorielle du moi de Dante :

D Ich bin lange gereist bin irgendwo angekommen hineingeschlafen [...] V (wird langsam beleuchtet. Rötlicher Schein. Er sitzt weit von D ab) / Ich bin Dante Alighieri ich bin zurückgekehrt in eine Stadt / aus der ich vor drei Jahrzehnten geflohen bin / Das Todesurteil war über mich verhängt worden / Zwanzig Jahre ist es her dass sie noch Macht hatten / mich durch den Kamin zu jagen. (Mü, 108). était une sorte de souffleur qui redonnait à Dante son nom et son passé49. «D Du 
sprichst von mir / du gibst mir einen Namen zurück / und eine Vergangenheit. » (Mü, $367)$, tandis que la version 3.1 opère une fusion complète : seules les indications « $\mathrm{D}$ » et « $\mathrm{V}$ » en marge d'une longue réplique indiquent une distribution possible à deux personnages, «V » demeurant par ailleurs « dans l'ombre ». Dans la version 3.4, Weiss revient au texte de la version 2. Dans la version 4 Virgile perd toute identité commune avec Dante et toute empathie pour devenir ce qu'il sera fondamentalement, mais avec plus de nuances, dans la version achevée : le sbire culturel du nouveau pouvoir de la cité qui le signifie non sans brutalité à un Dante encore désorienté - mais loquace et même prolixe quand il s'agit de qualifier une absence de trente ans non pas d'« émigration " mais d'« expulsion " - dans les deux brèves répliques du premier Chant: «Es ist Zeit. Ich bin gekommen, dich abzuholen. Hast du vergessen? /.../ Komm, sie warten auf dich »

Pour revenir à Dante, sa métamorphose de loque humaine - qui l'apparente à cet » homo sacer » décrit par Giorgio Agamben ${ }^{50}$ - en antagoniste définitif du pouvoir de ses anciens bourreaux à la fin de la version achevée d'Inferno, permettra à Weiss de lui conférer un grand nombre de caractéristiques qui ont valeur de biographèmes. Dans toutes les versions du texte, y compris dans la Dante-Prosa, il est montré assailli de doutes et de remords, d'un sentiment tardif de culpabilité, qui lui sera en permanence instillé par les puissants dès son retour dans sa ville natale, et faisant l'objet d'agressions physiques auxquelles le Dante de la Divine Comédie, d'abord menacé par le lynx, le lion et la louve, avait échappé. La grande déliquescence psychique et physique $\mathrm{du}$ «Heimkehrer» est très clairement mise en scène dans le «Canto 2 " de la "Aischmann-Fassung » où il décrit à Virgile la remontée du corps physiologique vers une conscience qui apprend peu à peu à articuler les mots et les pensées (Mü, p. 412-413). Dans Inferno et ses différentes versions, le mutisme d'un Dante incapable de comprendre la réalité qui l'entoure et de trouver les mots pour la dire, ses balbutiements et gargouillis que l'on retrouvera par endroits dans la version achevée de l'œuvre, sont surtout exhaussés au début de la version 7 qui dépersonnalise le protagoniste : les didascalies parlent d'un Dante devenu un locuteur à numéro («Spre 1 »), gisant sur la scène, comme "mortellement blessé », et qui ne peut que "gémir » ou émettre des "bruits de gorge " avant de "balbutier» devant les incitations des " voix » qui l'entourent: "Wie sprechen / wie Worte mehrmals gebrochen wiederholt" (Mü, p. 405).

C'est le « Giotto-Entwurf » qui montrera avec le plus de netteté, à l'instar de la version finale, la sortie de l'hébétement muet initial de Dante et sa résistance progressive au harcèlement des puissants qui préludera à la déprise finale clairement formulée au dernier Chant de la version achevée : là, soumis à un harcèlement qui prendra vers la fin de la pièce la forme symbolique d'un filet que l'on jette sur lui, Dante va progressivement à la fois accepter les conséquences individuelles de ce difficile et pénible travail de mémoire sur son passé et finir par refuser tout compromis avec un pouvoir qui ne lui laissait comme espace existentiel et artistique que les honneurs factices d'un poète complaisant et totalement muséifié. Le dernier Chant d'Inferno met néanmoins en scène le pessimisme historique foncier de Peter Weiss - le "chefpatron" s'apprête à appuyer sur un bouton qui montre que l'éternel retour de la guerre sera cette fois atomique - qui fait immédiatement suite à l'ultime sursaut subjectif d'un poète à la fois humilié et récupéré, bête de cirque et pièce de musée : «Ich sage mich / für immer / von euch los » ${ }^{51}$, avait dit Dante, tandis qu'il s'arrachait 
les attributs de sa pétrification allégorique, la couronne de laurier, dont Virgile venait de ceindre son front, et le bâton, attribut symbolique de ses pérégrinations.

Pour terminer, je proposerai quelques pistes de lecture pour le grand texte en prose auquel Müllender, à la suite de Jens Birkmeyer, donne le titre de « Dante-Prosa » - alors que le nom de Dante en est complètement absent - et qui peut être considéré comme la clôture du "Projet-Divine Comédie ». Ce texte réécrit les 12 premiers chants de la Divine Comédie en un bloc de prose qui ignore tout alinea, préfigurant la typographie compacte de Ästhetik des Widerstands que Weiss justifiait ainsi :

Es gehört für mich zur Ästhetik dieses Komplexes, dass der Ich-Erzähler mit großen Blöcken konfrontiert ist, die nicht zu teilen sind; es geschieht eben alles in einer ungeheuren Geschlossenheit ${ }^{52}$.

La Dante-Prosa se voulait elle aussi "aus einem Guss ", un bloc pour faire bloc: entre Peter Weiss et Dante, entre le dialogue théâtral et le monologue en prose, entre le sujet et son objet. Dans son ouvrage déjà mentionné, Jens Birkmeyer a le premier analysé la dimension autobiographique du texte: la maladie, la crise de l'écriture, l'hostilité croissante envers la critique, le sentiment de culpabilité tenace du « survivant » ou les conflits avec les puissants du « socialisme réellement existant ». Remarquant que c'est toujours dans les périodes de crise que s'opère le rapprochement avec Dante, Y. Müllender souligne que, de toutes les réécritures weissiennes de la Divine Comédie, c'est la Dante-Prosa qui présente le plus de références intertextuelles au texte de Dante (Mü, 297).

38 Le début du texte marque d'emblée - en mettant l'incipit de la Divine Comédie au conditionnel et en recourant à plusieurs prolepses - la distance avec le Je-narrateur de la Divine Comédie et la proximité immédiate de l'autobiographique :

Wäre ich schon in der Mitte meines Lebensweges hier angelangt, so hätte ich mir viele der späteren Verirrungen, Trugschlüsse und nutzlosen Tätigkeiten erspart. Jetzt aber war mein fünfzigstes Lebensjahr überschritten (Weiss avait 53 ans en 1969, A.C.) und Krankheiten, sowie immer stärker werdende Anfälle einer Müdigkeit, die ihren Grund in eben jenen Irrtümern und missglückten Anstrengungen, hinderten mich daran, die Entdeckungen dieser Reise vollauf auszunutzen, und mit ihnen der mir noch zur Verfügung stehenden Zeit einen neuen Sinn zu geben.

Les cinq années qui s'écoulèrent entre Inferno et ce sombre bilan autobiographique furent riches en déceptions, critiques agressives et combats ressentis souvent - les Notizbücher l'attestent - comme problématiques : représentations controversées de Die Ermittlung, critiques ouest-allemandes massives après la prise de partie pour le socialisme, dissensions avec le " socialisme réellement existant ", surtout après Prague, semi-échec de ses pièces directement politiques entre 1967 et 1969 (Gesang vom lusitanischen Popanz, Vietnam-Diskurs, Wie dem Herrn Mockinpott das Leiden ausgetrieben wird), polémique avec le Groupe 47 à Princeton en $1966^{53}$. En ce sens, la Dante-Prosa met essentiellement en scène un processus autoréflexif sur les activités littéraires et politiques de Peter Weiss dans la deuxième moitié des années soixante. Dante n'apparaît plus ici que comme possibilité littéraire du biographique dont est souligné d'entrée de jeu, sans euphémismes, le caractère fictionnel : impossible de travestir son propre vécu en s'aidant du grand récit épique dantesque, de sa fiction de base et de son décor initial :

Doch fällt es mir schon schwer, den Anfang zu beschreiben, denn es war kein Wald, auch keine Einöde, in die ich geriet, um alles Alte abzustreifen und mich der Erleuchtung auszusetzen, es war kein Meer des Wassers oder der Luft, kein 
Herausgerissensein aus allem Alltäglichen und Gewohnten, sondern das Waschzimmer eines städtischen Hauses, in dem ich ins Handbecken Blut pisste (Mü, 505). Chant de la Dante-Prosa en un je-narrateur inscrit dans une réalité autobiographique beaucoup plus concrète : celle d'une discussion publique - il s'agit de toute évidence d'une rencontre du "Groupe 47 » - où le je-narrateur désemparé, voire «oppressé » par la violence de ces attaques, a vu non seulement ses textes, mais aussi ses opinions et ses engagements massivement critiqués. L'allusion à la maladie - les coliques néphrétiques qui pourraient être un symptôme de somatisation ${ }^{54}$ - montre que l'identification fictionnelle de Peter Weiss à Dante va elle aussi se préciser : le lynx, la louve et le lion sont ici les masques métaphoriques de professionnels de l'éreintement critique qui vont - «dröhnend, laut und unversöhnlich » - tenter d'anéantir l'œuvre puis, à travers elle, l'auteur qui finira par tomber en syncope, abandonné par un public en qui il avait eu la faiblesse de placer ses espoirs. Au Chant 3, on retrouvera cette micro-société paradigmatique baignant dans une mondanité culturelle factice - décrite avec le grand luxe de détails que permet la prose descriptive -, flattant puis humiliant le je-narrateur avant de traverser l'Achéron dans la barque de Charon, celui-ci retrouvant sa figure mythologique (et dantesque) alors qu'il avait été, comme nous l'avons vu, l'un des masques du «chef/patron » d'Inferno. Le récit prend une tonalité particulièrement caustique qui en dit long sur l'irritation accumulée par Peter Weiss :

...hörte ich, wie sie sich über ihre Beute ausliessen, um ihre Überlegenheit kundzutun, um schnell in der Menge aufzuglänzen und dumme Augen, die sich auf sie richteten, zu blenden. Sie, die Nichtskönner, die Nullen, die Kraft ihrer Frechheit, ihrer Unverschämtheit, sich das uneingeschränkte Recht des Urteilens zugeschanzt hatten... (Mü, 512).

41 Le Chant 2 (Mü, 508-510) décrit, sur un mode plus ramassé, certaines des visions d'horreur disséminées dans les différentes versions d'Inferno. Le je-narrateur y apparaît terrassé par ce « qui n'a pas de forme » et « pas de substance » - une définition possible de l'angoisse absolue - et entouré de mourants semblables à des « amibes, des éponges ou des algues", recouverts de cette "boue faite de larmes, d'urine, de sang et d'excréments " qui pourrait être une fosse commune imaginaire et qui menace de l'engloutir. C'est une « communauté terrible avec ceux qui furent une fois pour toutes liquidés » à laquelle le je-narrateur devra impérativement donner forme langagière pour pouvoir commencer ses pérégrinations. On a affaire ici à des tableaux proprement infernaux, au sens des visions d'horreur des cercles de L'Enfer de Dante : ils réitèrent aussi les hallucinations de l'un des fous de Marat/Sade ${ }^{55}$ et font retour sur les fantasmes et obsessions des tableaux de jeunesse de Weiss qui étaient autant de métaphores de son sentiment existentiel ${ }^{56}$. Dans ce Chant, plus que dans tout autre, la description de l'horreur évoque le principe d'anesthésie qui sera thématisé dans Die Ästhetik des Widerstands et dont la mise en pratique est la condition subjective d'un art engagé dont il préserve le pouvoir de subversion :

Die Anästhesie gehöre auch zur äußerst beteiligten, Stellung beziehenden Kunst, denn ohne deren Hilfe würden wir entweder vom Mitgefühl für die Qualen andrer oder vom Leiden am selbsterfahrenen Unheil überwältigt werden und könnten unser Verstummen, unsre Schreckenslähmung nicht umwandeln in jene Aggressivität, die notwendig ist, um die Ursachen des Alpdrucks zu beseitigen ${ }^{57}$.

Ce Chant lie avec force les réminiscences de la Shoah et du nazisme au « Kulturbetrieb » d'un présent qui décalque celui de la République Fédérale des années soixante (aux 
Chants 1 et 3) et dont certaines institutions culturelles semblent se faire un malin plaisir d'accabler, avec apparemment plus d'égards que le "chef-patron " au début d' Inferno, un je-narrateur qui fut jadis «condamné à mourir suffoqué par la fumée ». Au même moment, sur une place proche, se terminent les préparatifs d'une nouvelle «Bücherverbrennung » qui peut figurer ici la métaphore la plus signifiante des avanies subites au Chant suivant par ce même je-narrateur et que le je biographique vécut, à l'instar d'un Celan, comme une tentative de liquidation symbolique.

$\mathrm{Au}$ fil des Chants de la Dante-Prosa est dessinée une vision cauchemardesque d'un totalitarisme impitoyable où se rejoignent l'Enfer de Dante, les réminiscences du «Troisième Reich » et les discours sur l'État-policier répressif et fascistoïde de la fin des années soixante en RFA: ainsi le Chant 5 décrit-il, avec quelques réminiscences kafkaïennes, un appareil judiciaire totalitaire et régalien dont les fonctionnaires déploient une activité à la fois fébrile et improductive, sous la haute autorité du juge Minos qui condamne par fournées entières des luxurieux et incestueux envers lesquels il est strictement interdit de manifester une quelconque pitié. Ce totalitarisme, qui transcende les régimes historiques pour s'imposer comme structure socio-politique dominante $\mathrm{du} \mathrm{xx}^{\mathrm{e}}$ siècle, découpe, plus nettement que dans Inferno, la ville de Dis en blocs représentatifs des hiérarchies de la société occidentale des années soixante telle que Weiss la percevait : les puissants assoient leur pouvoir sur l'aliénation apathique des dominés aux valeurs d'une société de consommation effrénée et sans entraves et soumettent à une terreur policière implacable les exclus définitifs de cette nouvelle mouture capitaliste de la "Volksgemeinschaft». Cité rouge de sang du bas-enfer où veillent les antiques Furies et la Gorgone/Méduse dans la Divine Comédie, la ville était devenue au Chant 4 d'Inferno la ville lisse et apaisée, "d'où est absente toute douleur " et où l'argent-roi est le nerf du pouvoir comme de la culture, bref, une métaphore vraisemblable de la restauration ouest-allemande et de son « miracle économique ». Par un détournement cynique d'une vision utopique de Dante - «Städte will ich mir denken /.../ in denen du nicht bei jedem Schritt / Ausschau hälst / nach einem Seitenweg zur Flucht »- les séides du pouvoir pouvaient, selon le rêve de leurs maîtres, la présenter comme la "ville de la paix » (Chant 23). Dans la Dante-Prosa elle ne pénètre que tardivement - tout à la fin du Chant 8 - la conscience d'un je-narrateur qui l'avait vécue jusqu'alors comme une ville des tourments toujours renouvelés avant de la percevoir au Chant suivant comme une ville ultramoderne à l'activité débordante et aux parades militaires martiales : tandis qu'il se décrit subissant un interrogatoire de nouveaux SS («schwarze knisternde Kleidung, Stiefel, Helme mit Sturmriemen, Revolvertaschen ») et se reproche de ne tirer aucun désir de résistance de son infériorité58 on y entend d'innombrables haut-parleurs retransmettre le discours d'une voix qui semble émaner de la statue dorée d'un hybride dignitaire nazi : «eine gigantische Figur, aus gleissendem Gold, hoch aufgereckt das Gesicht, weit aufgerissen der Mund und die Augen, [...] eine Hand in geöffnetem Griff erhoben, die andre Hand gekrallt in die vorschwellende Brust, der Leib trächtig, breit gespreizt die Beine ». Il est significatif que, dans ce même Chant, en réponse au discours hystérique sans contenu qui fige les corps uniformisés des sbires du pouvoir, ce soit Phlegias - ancien souffredouleur du je-narrateur dans la Dante-Prosa mais sorte de double d'Hitler dans les violents discours antisémites et rassistes que le même personnage prononçait au Chant 13 d'Inferno $^{59}$ - qui dresse le portrait intellectuel, politique, artistique et social le plus proche du Dante historique et d'un idéal du moi de Peter Weiss (Mü, 530-531). La réaction du je-narrateur est tout aussi significative: son monologue intérieur n'y 
perçoit qu'une louange imméritée qui lui sert peut-être de sauf-conduit mais fait surtout de lui un "hypocrite, transfuge et lèche-botte » dont les fantasmes de discours libertaire (toujours en monologue intérieur) masquent mal une non résistance de fait aux excès du pouvoir. Cette réaction est très probablement révélatrice de la situation psychique de l'auteur de la Dante-Prosa à la fin des années soixante et partant du contexte personnel de sa relecture de la figure du poète italien: entre une autodépréciation qui intériorise des critiques pourtant ressenties comme infâmes et les accès dépressifs chroniques de la deuxième moitié des années soixante il n'y avait, ni objectivement ni subjectivement, pas de place pour un « héros positif ».

$\mathrm{Au}$ fond, ce Dante-là " angefressen von der Zweifel-Krankheit », comme Weiss luimême, pourrait être un habitant typique du "Purgatorio » et on comprend que peu avant sa mort ce dernier ait eu le projet d'écrire une pièce centrée sur cette topographie-là̀ ${ }^{\circ}$.

Le «Purgatorio », nous dit la Vorübung, est le lieu des errements et de l'échec, du doute et de la pusillanimité, mais aussi celui du mouvement et de la possibilité d'une pensée du changement au milieu des multiples exhibitions de la société marchande du spectacle, du « grand marché, traversé des rugissements des slogans et de la respiration brûlante de la concurrence » :

...die Gegend des Zweifelns, des Irrens, der mißglückten / Bemühungen, die Gegend des Wankelmuts und des ewigen Zwiespalts, doch immerhin / gibt es hier die Bewegung, es gibt den Gedanken an eine Veränderung / der Lage, selbst wenn es unmöglich scheint, den Wulst / zu durchbrechen, der jede unsrer Regungen einengt ${ }^{62}$.

C'est le lieu éminemment humain de l'hésitation timorée mais aussi du combat et de l'espoir du nouveau, « lieu de l'entre-deux » qui horrifie le thuriféraire de l'ordre établi qu'était devenu le Virgile d'Inferno : «Irgendwo gibt es den Zwischenzustand / den eine unbekannte Ordnung Purgatorio nennt / Schrecklich muss es da sein / da solls nur Wankelmut und Zweifel geben... » (Chant 17 de Inferno). C'est un lieu où «l'attention est démoralisée " et "l'effort de survivre toujours plus difficile", lieu éminemment productif du paradoxe et de la contradiction, de l'affaissement comme du redressement, où on " annonce la couleur » et où « on pose les questions qui exigent une décision». C'est dans cette logique que, dans le Gespräch über Dante, "A »-Peter Weiss motive sa volonté de réécriture de la figure de Dante: «Wieviel Irrungen, Zweifel, Begierden, Befürchtungen und Hoffnungen. Diesen Dante kann ich in meine heutige Welt aufnehmen ».

Notons aussi qu'étymologiquement, le Purgatoire est le lieu de la purge, celui où on purge une peine qui purifie - le Dante de la Divine Comédie parle d'ailleurs du Purgatoire comme de la «montagne de la purification »-, c'est donc aussi peut-être le lieu de la catharsis du théâtre aristotélicien. Un lieu à revisiter, à réhabiter et peut-être à réhabiliter dans l'imaginaire occidental, car il est pleinement la scène principale de l'ici-bas : de son agencement dépend la possibilité de ne pas tomber dans l'un des deux abîmes - «Inferno » et «Paradiso "-, dont Peter Weiss avait voulu montrer qu'ils étaient les faces pérennes, à la fois visibles et cachées, de l'après Shoah ${ }^{63}$.

Mais la Dante-Prosa met aussi en scène une autre figure de Dante, ce en quoi Müllender (Mü, 290 sq.) a raison d'y percevoir une modification profonde de la topologie initiale : Dante n'est plus seulement, comme dans Inferno, le «Heimkehrer» rescapé de l'extermination qui revient dans une ville devenu le paradis des bourreaux de jadis, 
préoccupés au premier chef de récupérer l'ancien poète en lui rappelant sa participation à d'anciennes persécutions qui préfiguraient bien d'autres tourments à venir, disqualifiant par là son statut potentiel de symbole de la dissidence. Il est aussi l'ancien résistant clandestin confronté à un pouvoir répressif et à la trahison parfaitement assumée de ses anciens compagnons de lutte devenus eux-mêmes de zélés bourreaux. Cela est manifeste dans la description des rencontres avec les personnages dantesques de Ciacco (Chant 6), Farinata ou Cavalcante (Chant 10) qui ne sont plus des amis de jeunesse, en partie autobiographiques, abandonnés par Dante/Peter Weiss mais des traîtres à l'ancienne cause ayant fait allégeance au pouvoir. Ceci vaut tout particulièrement pour le discours de Ciacco dans la scène du lupanar au Chant 6 (Mü, 521-522) : sur fond de "gémissements de plaisir ", le membre " pétri » par une femme qui ne suscite que son "indifférence ", celui-ci rappelle à Dante que les luttes passées n'avaient enseigné qu'une chose, à savoir l'infériorité définitive des rebelles qui leur faisait devoir de s'attirer les bonnes grâces du pouvoir détesté en trahissant et torturant les anciens compagnons de lutte. Ce discours littéralement ob-scène rappelle le discours de Sade sous le fouet dans Marat/Sade, à la différence que le plaisir sensuel recherché dans la soumission des victimes se nourrit de la réminiscence des anciens tourments d'abord subis avant d'être infligés à des résistants :

Dass wir selbst die Gefängnisse kennen, dass wir selbst gepeitscht und mit glühenden Eisen versengt wurden, steigert unsern Spass [...]. Wir haben durch unsere Erfahrungen von Schrecken jede Zurückhaltung verloren (Mü, 522).

Comme le souligne Müllender, le rappel d'ignominies passées ne doit pas ici servir à nier l'activité de résistant mais à la placer après-coup sous le signe d'idéaux frelatés qu'il convient d'abjurer pour rentrer dans le giron de la société des puissants :

Starke Betonung findet die willenlose Subordination der Unterdrückten, deren passiver Gehorsam die bestehenden Ungerechtigkeiten und das Machtmonopol einer privilegierten Elite aufrechterhält (Mü, 282).

50 Le Chant 7 , qui voit Virgile défendre pour la première fois le pouvoir et l'ordre des exploiteurs et oppresseurs - le " grand ordre $»^{64}$ que Dante critique ouvertement et qui sera lié au "Betrieb des Wettbewerbs" capitaliste au Chant 12 - va, par opposition, dans le sens d'une plus grande importance accordée à la dissidence de Dante et à sa révolte contre l'ordre établi de la cité qui ne s'exprimait vraiment qu'au tout dernier Chant (33) de la pièce de 1964. Cet ordre établi, qui était affirmation d'une adéquation posée comme intangible entre le désir du plus grand nombre de respecter le «grand ordre » de l'accroissement et de la consommation des richesses et leur passivité politique, a ici pour emblématique repoussoir le gigantesque esclave aveugle et affreusement mutilé Pluto qui semble tout droit sorti des descriptions du «PergamonAltar » au début de Die Ästhetik des Widerstands alors qu'il avait pu être, nous l'avons vu, le symbole de la "ploutocratie " au pouvoir : une manière pour Peter Weiss d'établir, comme pour Phlegias et d'autres personnages, un intertexte différencié voire contradictoire à l'intérieur de sa propre œuvre :

Diesem Sklaven hier, den wir Pluto nennen, haben wir alles das genommen, was wir jetzt selbst empfinden wollen, die Augenfreude, das Tastgefühl, die wohlige Lässigkeit, mit der wir unsere Worte formen. Dieser verstümmelte Leib, den wir hier aufgestellt haben, dieser entmenschte Riese, dessen Schädel zertrümmert, dessen Geschlecht abgeschnitten ist, soll uns die Gaben nur noch wahrnehmbarer machen (Mü, 523).

51 On peut voir là la mise en scène d'une ultime perversion d'une société totalitaire qui, comme le montre le Chant 7 , enfonce dans la boue sur laquelle elle est édifiée les 
innombrables opprimés qui essaient de surnager et d'implorer une improbable pitié, comme cette femme au crâne rasé, motif récurrent de la Dante-Prosa, qui s'accroche aux jambes de Dante avant d'être brutalement piétinée par Virgile. Ne pas reconnaitre que l'on marche à chaque pas sur ce peuple de la fange, ces « Namenlose » dit Virgile, c'est se «tromper soi-même » (Mü, 523-524). Le Chant donne à tous les opprimés de la ville de Dis les traits les plus caractéristiques des victimes de la Shoah, les constituant en figuration archétypale de l'oppression la plus féroce. Il exhausse en même temps, dans une scène de chambre de torture, l'une des dimensions profondes du sadisme qu'avait pu pointer le Marat/Sade: la naissance du plaisir esthétique dans la jouissance des tortionnaires (Mü, 525). Ce Chant est aussi celui où une petite phrase de Dante, qui deviendra l'un des topos principaux de Die Ästhetik des Widerstands, provoque significativement la colère muette mais menaçante de Virgile :

Und habt doch Furcht vor denen, sagte ich, denen gegenüber ihr eure Macht ausübt, und geht doch nur unaufhörlich gegen sie an, weil ihr keinen Atemzug lang vor ihnen sicher seid (ibid.).

Il pointe, au détour d'une phrase, la grande peur des dominants - si totalitaire que soit leur domination - devant toute révolte ou révolution que ne manque pas de provoquer la cruauté croissante de leurs répressions. L'avant-dernier Chant (11) soulignera à quel point celles-ci sont une réponse anticipée à une "tempête » voire un "ouragan » redoutés et donc un aveu d'angoisse permanente, comme l'énonce Virgile, à l'aide de dénégations révélatrices, à un je-narrateur auquel Weiss, préparant le terrain idéologique à Die Ästhetik des Widerstands, semble laisser le dernier mot :

Die Sicherheit, das ungeheuer fest Gefügte dieser Stadt, ruht auf der nie erwähnten Möglichkeit, dass sie einmal an irgendeinem Punkt untergraben werden könnte, dass sich einmal im polierten Boden ein Loch auftun könnte oder ein Riss, in dem plötzlich das eben noch Gegenwärtige versackt. Für niemanden aber ist dies ein Grund zur Beunruhigung, die Aussicht auf einen solchen Wegfall ist so gering, dass er in keinem Plan erwogen wird. Und doch, sagte ich, ist alles, was hier geschieht, nur Antwort auf einen Sturm, auf einen Orkan, der kommen könnte, der alles niederreisst und wegfegt (Mü, 536-537).

\section{NOTES}

1. 1963 : Nacht mit Gästen - Marat/Sade, 1964: Inferno (publié en 2003), 1965: Die Ermittlung, 1966: Gesang vom lusitanischen Popanz, 1968: Viet Nam Diskurs - Wie dem Herrn Mockinpott das Leiden ausgetrieben wurde, 1970: Trotzki im Exil, 1971: Die Versicherung - Hölderlin.

2. Cette formule fait allusion au texte de septembre 1965, 10 Arbeitspunkte des Autors in einer geteilten Welt, où Weiss manifeste son adhésion aux principes du socialisme («Die Richtlinien des Sozialismus enthalten für mich die gültige Wahrheit») dans un monde marqué par les antagonismes de la guerre froide (cf. Peter Weiss, Rapporte 2, Suhrkamp, Frankfurt am Main 1971, pp. 14-23).

3. Signalons ici, avant d'y revenir, les deux principaux textes qui préparèrent et accompagnèrent le projet: Gespräch über Dante, rédigé parallèlement au travail sur Paradiso/Die Ermittlung (printemps 1965), a été repris, avec celui de la Vorübung zum dreiteiligen Drama divina commedia (= 
Vorübung), d'abord publié dans Akzente en 1965 mais dont la rédaction est contemporaine de celle d'Inferno (fin 1964), in : Peter Weiss, Rapporte, Frankfurt/Main, Suhrkamp, 1968.

4. « Es zeigte sich (in der Divina Commedia) nicht nur der Pfad hinein in das Seelengebäude des Inferno, in dem das Rohmaterial einer Epoche sich zu subjektiver Vision verdichtete, sondern der Schritt in den Mechanismus der künstlerischen Arbeit » (Die Ästhetik des Widerstands, Suhrkamp, Frankfurt am Main, 1988, p. 80).

5. Je n'approfondirai pas davantage cette thématique assez bien connue, me contentant de renvoyer au travail très documenté de Thomas Taterka, Dante Deutsch. Studien zur Lagerliteratur, Berlin, Erich Schmidt 1999, qui critique sévèrement le texte de Walser. Sur la culture de la mémoire et la littérature de témoignage sur les camps d'extermination, on se reportera avec profit à : Ulrich Baer (Hg.), "Niemand zeugt für den Zeugen ». Erinnerungskultur nach der Shoah, Suhrkamp, Frankfurt am Main, 2000.

6. Cf. P. Weiss, Die Ermittlung, Suhrkamp, Frankfurt am Main 1991 - P. Weiss, Inferno Stück und Materialien. Suhrkamp, Frankfurt am Main 2003, qui reprend la seule version achevée de ce texte, de décembre 1964.

Il faut également citer ici deux ouvrages fondamentaux récents qui proposent une analyse détaillée du " projet Divine Comédie »:

- Christoph Weiss: Auschwitz in der geteilten WeltPeter Weiss und die 'Ermittlung' im Kalten Krieg, St. Ingbert, Röhrig Universitätsverlag, 2000.

- Yannick Müllender: Peter Weiss' Divina Commedia-Projekt 1964-1969. 'läßt sich dies noch beschreiben' Prozesse der Selbstverständigung und der Gesellschaftskritik, St. Ingbert, Röhrig Universitätsverlag, 2007.

Il faudrait y ajouter le Peter Weiss Jahrbuch 6, Opladen/Wiesbaden, Westdeutscher Verlag, 1997, qui fait le point sur ce projet, et l'ouvrage plus ancien de Jens Birkmeyer, Bilder des Schreckens. Dantes Spuren und die Mythosrezeption in Peter Weiss' Roman "Die Ästhetik des Widerstands", Deutscher Universitäts-Verlag, Wiesbaden 1994.

Dans un article à paraître, j'ai analysé la version publiée d'Inferno dans le contexte du «projet Divine Comédie ». J'en reprendrai ici certains développements en intégrant les témoins de genèse publiés dans de très substantielles annexes par Y. Müllender sous la forme de versions numérotées de 0 (Arbeitsschema) à 11. Ces annexes contiennent également le grand texte inachevé en prose de 1969, que l'on appelle Dante-Prosa, rédigé entre la fin août et la fin septembre 1969, et qui marque une sorte de changement de paradigme intellectuel et littéraire du projet avec un retour signifiant à la prose narrative. Les citations de ces textes reproduiront la graphie de Peter Weiss reprise par Müllender. Elles seront suivies de la seule indication Mü $+\mathrm{N}^{\circ}$ de page.

7. Braun et Unseld avaient trouvé, contrairement au Marat/Sade, Inferno peu compréhensible pour un public allemand et international, ce qui avait amené Weiss à détacher Paradiso de la trilogie pour le publier sous un autre titre et le faire jouer dans l'urgence de la conjoncture de la fin des procès d'Auschwitz (été 1965), sur lesquels je reviendrai. Unseld lui avait conseillé de gommer dans le texte - titre, divisions en Chants - toute référence à la Divine Comédie, tout "habillage dantesque», ce que Weiss accepta en grande partie. Voir là-dessus : Y. Müllender, Peter Weiss' "Divina Commedia »-Projekt, op. cit., p. 199 sq.

8. Weiss collaborera directement à celle de Piscator à la Freie Volksbühne de Berlin-ouest. Cf. Denis Bablet, «L'Instruction de Peter Weiss», in : Les voies de la création théâtrale II, études réunies par D. Bablet, Paris, Ed. du CNRS, 1970, p. 157-235.

9. Comme dans les écrits autofictionnels Abschied von den Eltern (1961) et Fluchtpunkt (1962), les journaux de Paris et de Copenhague (1960-1961) ou dans le «journal de travail» que sont les Notizbücher, certes nettement plus subjectifs que les Arbeitsjournale de Brecht qui les inspirèrent.

10. Cf. Inferno (Chant 6), p. 26. Cf. aussi le «Canto 2 » de la version 8 : «Erst als das Bewusstsein allmählich zurückkam, dachte ich, dass so das Sterben sein müsse, der letzte Augenblick, in dem 
es die Welt noch gibt, aber nicht mehr das Erkennen» (Mü, p. 412). Les différentes versions du texte insisteront sur ces longs balbutiements, ce «Dämmerzustand» de la conscience du sujet retour d'exil, semblable à celle d'une "amibe", comme Dante le dit encore dans une longue tirade qui ouvre le Chant 27 de la version achevée : « O die Begrenztheit / dieser Dämmerzustand / dieses niedere Dasein / Ich weiss / dass es ein Verstehen gibt / manchmal bin ich nah daran / dann bin ich wieder dort / wo die Amöbe ist » (p. 95).

11. Je citerai ici un passage de la « Aischmann-Fassung » d'Inferno: « Ich folgte mit als Möbelstück in einem Haushalt / ich liess mich verfrachten in ein anderes Land / ohne zu verstehn dass dies meine Rettung war » (Mü, 439).

12. Comme pour Dante, la figure de Béatrice qui prendra la relève de Virgile pour accompagner le je-narrateur au paradis, est un personnage fictionnalisé qui est au croisement du biographique et de l'esthétique. Pour Weiss, elle sera par le truchement du Dante d'Inferno la figure de la mauvaise conscience par excellence. Le Gespräch über Dante fera de la trahison envers Béatrice une trahison envers la pratique poétique : «Ich wurde vertrieben, geriet ins Exil. Beatrice blieb drüben [...]. Hätte ich Mut gehabt, hätte ich sie auf die Flucht mitgenommen [...] ? Beatrice kam um. Vielleicht wurde sie erschlagen. Vielleicht vergast. Sie war längst zu Asche geworden, da beschrieb ich mir noch ihre Schönheit. (souligné par moi, A.C.) », (op. cit., p. 154).

La version achevée d'Inferno qui fera de Béatrice/Bea une "matrone digne ", "veuve de soldat» et "épouse d'un directeur de banque " (Chant 3) donnera néanmoins l'occasion aux représentants du pouvoir, qui pointent sans relâche la couardise passée du "Heimkehrer », de discréditer la poésie amoureuse de Dante : «Um den Verlust der Bea hat er wohl geklagt / doch war sie ihm zum Träumen und zum Beten gut / mit ihr zu leben hätte er doch nie gewagt / nur im Gedichte hatte er zur Liebe Mut». Procédé récurrent dans tout le texte : c'est le pouvoir restauré des anciens bourreaux qui impose à Dante, ici proche du moi biographique de Weiss, de réinterroger son exil et les motivations profondes de sa pratique poétique. La Dante-Prosa transformera les reproches extériorisés des versions théâtrales en reproches intériorisés dans de longs monologues intérieurs (Chant 5). Dans le deuxième Chant de ce texte, c'est une Béatrice horriblement mutilée - «die Brüste verschrumpft, Arme und Beine dünne verschorfte Stöcke, das Kinn hochgeworfen, der Mund ein offenes Loch, die Nase abgeschlagen, das Haar abgeschoren, stumpf die gebrochenen Augen » - qui sera, avec d'autres femmes assassinées par les nazis, le catalyseur d'une appartenance fantasmée, martelée par l'épiphore identitaire à double sens „ich weiss“, au peuple de la Shoah : « ja, schrie ich, ich weiss, ich lag unter ihnen, ich weiss, ich hätte unter ihnen liegen können, ich gehörte zu ihnen, ich bin entflohen, entkommen, willst du mich denn wieder dorthin treiben » (Mü, 509).

13. Gespräch mit Dante, op. cit. p. 148.

14. P. Weiss, Die Ästhetik des Widerstands, op. cit., p. 83.

15. Pour Peter Weiss comme pour Hannah Arendt ces procès furent, par-delà la re-présentation du lieu le plus emblématique de la Shoah, autant de révélations sur l'état des consciences ouestallemandes confrontées au retour du refoulé. Comme elle, Peter Weiss assista à plusieurs audiences du procès - surtout à l'automne 1964, lors de la rédaction d'Inferno - tout en lisant régulièrement les comptes-rendus de Bernd Neumann qui seront la base documentaire principale de Die Ermittlung (Cf. Bernd Naumann, Auschwitz. Bericht über die Strafsache gegen Mulka und andere vor dem Schwurgericht Frankfurt, Suhrkamp, Frankfurt am Main 1968. Cf. également le dernier texte sur le national-socialisme écrit par H. Arendt, "Der Auschwitz-Prozess ", préface de l'édition américaine du livre de Neumann publié en traduction américaine en 1966. Le texte a été réédité in: Hannah Arendt, Nach Auschwitz. Essays \& Kommentare 1, Berlin, Thiamat 1989, p. 99-136).

Sur la bibliographie accumulée par Peter Weiss lors du travail sur Die Ermittlung, on se reportera à l'ouvrage déjà mentionné de C. Weiß, Auschwitz in der geteilten Welt, op. cit., p. 92-94. On y trouve le 
livre de Primo Levi, Ist das ein Mensch? (Si c'est un homme ?) dont on sait qu'il souligne l'importance qu'eut la Divine Comédie pour sa survie intellectuelle.

16. Cf. Y. Müllender, Peter Weiss' « Divina Commedia »-Projekt, op. cit., p. 197.

17. Dans cet « Aischmann-Entwurf» le substitut de Dante prend le nom d'un futur compagnon de lutte antifasciste du Je-narrateur de Die Ästhetik des Widerstands (avec une graphie légèrement différente, «Ayschmann »). C'est un jeune communiste avec lequel celui-ci commentera les chefs d'œuvre de l'art mondial : du « Pergamon-Altar » à Guernica de Picassoen passant par Le Radeau de la Méduse de Géricault.

Il y a dans les trois premiers chants (1,2 et 4) - la version en comporte sept - des tirades bien plus longues de Dante-Aischmann que dans la version achevée. C'est un long récit en prose à la première personne à peine interrompu par les questions ou remarques de Virgile (devenu Gil) (Chants 1 et 2) ou de Charon et de ses « collaborateurs » (Chant 4). La syntaxe y est hypotactique, le mouvement lent et un peu lourd, ce que manifeste par opposition une deuxième version de ce Chant 4 (version 8.2) qui se rapproche de la forme en vers libres et répartit sur un seul vers principales ou subordonnées afin à la fois de concentrer et de rythmer l'expression. Aischmann est la version la plus politisée du signifiant « Dante ».

18. « Meine Ortschaft », in : Peter Weiss, Rapporte, Suhrkamp, Frankfurt am M. 1968, p. 116.

19. Un passage du schéma de travail initial d'Inferno (Chant 16) montre sans équivoque, et avec une grande concentration de sens que les différentes versions ne reprendront pas, l'échec non dénué d'ambivalences de la symbiose judéo-allemande : «haben wir nicht in euerm land mit gelebt euer schicksal zum eigenen gemacht eure sprache gesprochen so furchtbar tief mit eurer sprache verwurzelt in eurer sprache geträumt [...] haben wir nicht alles was wir wissen in eurer sprache empfangen / nannten wir uns nicht deutsche / mit den verworfensten Mitteln wurden wir von euch ausgesondert wir konnten es nicht verstehen hätten mitgemacht viele von uns / erkannten wir euch nicht? / PLÖTZLICH MUSSTEN WIR EUCH ERKENNEN SAHEN EUCH WIE IHR SEID / VERGEBEN KÖNNEN WIR EUCH NICHT UNSER IST NUR DIE GRENZENLOSE UNAUSLÖSCHLICHE TRAUER » (Mü, p. 352).

C'est surtout la version 8 d'Inferno qui reviendra sur la réappropriation d'une judéité éminemment complexe et a priori non-conforme à la «loi juive": la mère de Weiss était protestante et son père, qui était lui d'origine juive, s'était converti au protestantisme. Ce sera aussi une redéfinition après-coup de son émigration dans le sens de l'exil : « Aischmann Nennen wir diese Emigration Flucht oder Vertreibung. Von Entschluss konnte bei mir keine Rede sein. Es stellte sich plötzlich heraus, dass mein Vater einem Menschentyp angehörte der, infolge einer Besessenheit, die sich Rassenforschung nennt, Jude genannt wurde » (Mü, p. 417-418). Dans la version 10 d'Inferno, Dante reprend un biographème fréquent de Weiss et martèle face à Phlegias - «Chef der Universität» qui deviendra une figure du pouvoir nazi dans la version achevée qu'il ne fut pas «banni en tant qu'adversaire de la ville » : «Es ist wahr, als ich die Stadt verliess, mit der Familie, der ich angehörte, und mit den andern allen, die noch die Möglichkeit besassen, sich eine Ausfahrt zu erkaufen, war ich kein Feind, ich war nur ein Enttäuschter und Betrogner, zu keinem Kampf bereit, nur fassungslos. » (Mü. p. 463).

20. Vorübung, op. cit., p. 135.

21. «Unaufhörlich produzierten wir weiter, während draussen eine Welt in Stücke fiel. Der Krieg öffnete meine Augen nicht. Der missglückte Kampf um meine Berufung hatte mich in einen Zustand der Umnachtung versetzt. [...] Die Emigration hatte mich nichts gelehrt. Die Emigration war für mich nur die Bestätigung einer Unzugehörigkeit, die ich von frühester Kindheit an erfahren hatte. ", écrit-il dans les dernières pages de son premier écrit autofictionnel, Abschied von den Eltern ( Suhrkamp, Frankfurt am Main, 1961, p. 143).

22. Dans Fluchtpunkt Peter Weiss décrit ainsi cette première prise de contact avec l'univers des camps d'extermination qui provoque après coup le sentiment traumatisant d'une identité trouble et conforte le "syndrome du survivant »: "Auf der blendend hellen Bildfläche sah ich die 
Stätten, für die ich bestimmt war, die Gestalten, zu denen ich hätte gehören sollen» / «...wir sahen es (Unvorstellbares) in seinen Ausmaßen, die so ungeheuerlich waren, dass wir sie zu unsern Lebzeiten nie bewältigen würden» / «Dies war eine Welt, die von Menschen errichtet worden war. Und dann sahen wir sie, die Wächter dieser Welt, sie trugen keine Hörner, keine Schwänze, sie trugen Uniformen, und geängstigt scharten sie sich zusammen und mussten die Toten zu den Massengräbern tragen. Zu wem gehörte ich jetzt, als Lebender, als Überlebender, gehörte ich wirklich zu jenen, die mich anstarrten mit ihren übergroßen Augen, und die ich längst verraten hatte, gehörte ich nicht eher zu den Mördern und Henkern.» (Fluchtpunkt, Suhrkamp, Frankfurt am Main 1965, pp. 135 et 210).

23. Cf. Gerlach, Rainer / Richter, Matthias (Hrsg.): Peter Weiss im Gespräch, Suhrkamp, Frankfurt am Main 1986, p. 219.

24. Je renvoie aux analyses de ces deux textes, in: Rolf D. Krause: Faschismus als Theorie und Erfahrung. "Die Ermittlung » und ihr Autor Peter Weiss, Peter Lang, Frankfurt am Main/Bern 1982, p. $482 \mathrm{sq}$.

Il faut y ajouter les Notizbücher 1960-71, Frankfurt am Main, Suhrkamp 1982. Ce sont les Notizbücher $5(1.1 .64-26.4 .64)$ et $6(27.4 .64-12.7 .64)$ qui contiennent le plus de notes sur Inferno et les Notizbücher 17 et 18 (Digitale Bibliothek, voir n.1) sur la « Dante-Prosa ».

25. Y. Müllender: Peter Weiss' «Divina Commedia »-Projekt, op. cit., p. 207.

26. Cf. Peter Weiss: Notizbücher1960-71, op. cit., p.374. La critique anticapitaliste et antiimpérialiste, qui devient dominante chez Weiss à partir de 1965, est le maillon de la chaîne idéologique et sociale qui relie le nazisme à la RFA. On voit dans le Aischmann-Entwurf de Inferno comment l'anticommunisme triomphant en RFA est inscrit dans le sillage idéologique de l'antisémitisme nazi.

27. Cf. Fluchtpunkt, op. cit., p. 11: « Deutlich sah ich [...], dass ich auf der Seite der Verfolgten und Henker stehen konnte. Ich hatte das Zeug in mir, an einer Exekution teilzunehmen.» (voir aussi les Chants 12, 13, 15 et 31 d'Inferno qui placent les aveux de Dante sous le signe de cet harcèlement permanent par les anciens bourreaux préoccupés de le discréditer, de démontrer qu'il est un des leurs pour justifier sa réintégration / récupération). Dans une interview de 1965 Peter Weiss fait part de sa « chance » d'avoir été préservé par ses origine juives de servir dans la Wehrmacht et d'avoir été épargné d'une « complicité dans les crimes de guerres ».

28. P. Weiss: Die Ermittlung, op. cit., pp. 78-79.

29. Cf. l'interview d'octobre 1965 cité in : Y. Müllender: Peter Weiss' «Divina Commedia »-Projekt, op. cit., p. 221.

30. Th. W. Adorno: Negative Dialektik, cité in: Robert Cohen: «Identitätspolitik als politische Ästhetik. Peter Weiss' Ermittlung im amerikanischen Holocaust-Diskurs », in: Ulrich Baer: "Niemand zeugt für den Zeugen »... op. cit., p. 166. L'article de R. Cohen détaille les critiques virulentes adressées à Die Ermittlung par nombre d'intellectuels juifs américains qui reprochaient l'absence des termes «juifs» (remplacé par «Menschen») et «Auschwitz» (désigné par «Die Ortschaft ») ainsi que l'absence d'une catharsis de type aristotélicien dans le fonctionnement du texte théâtral.

31. Dans le texte à paraître déjà évoqué, j'ai souligné la rebellion contre le texte-source que peut être le dialogue intertextuel à l'œuvre dans la réécriture. Peu avant sa mort Peter Weiss y revenait: « ...auch das, was aus vergangenen Zivilisationen und Kulturen übernommen wird, wird von mir gegen den Strich gelesen. Es ist also weniger ,Erbe' als ,Neuschöpfung'. Es darf nicht übernommen werden wie etwas Hohes, Heiliges, sondern muss mit unseren eigenen Kräften neu gebaut werden, mit aller Anerkennung des einmal Geleisteten, doch immer wieder unter neue Vorzeichen gesetzt. » (Lettre de Peter Weiss à Manfred Haiduk du 17 mars 1982, citée d'après : Sture Packalén : «Peter Weiss und Martin Buber » in : Peter Weiss-Jahrbuch Bd. 5, Westdeutscher Verlag, Opladen 1996, p. 161-162). 
32. Nombre de commentateurs, dont Erich Auerbach dans son Dante und die irdische Welt, ont insisté sur le fait que «dans l'au-delà de la Comédie était contenu le monde terrestre » (Cf. Y. Müllender: Peter Weiss' «Divina Commedia »-Projekt, op. cit., p. 57). Dans Die Ästhetik des Widerstands, le je-narrateur et son petit collectif d'amis ne se laisseront pas rebuter par les difficultés de lecture initiales d'un univers historique visionnaire, apparemment si éloigné de leurs préoccupations immédiates, pour en apprécier le réalisme intrinsèque : «...und wenn es anfangs schien, als verberge sich diese Transformierung unter Symbolen und Allegorien, die nur dem verständlich sein konnten, der mit der Scholastik vertraut war, so ließ sich das Filigranwerk der Gleichnisse doch mehr und mehr abtasten nach Einzelheiten, die von einer aus unmittelbarer Nähe beobachteten Wirklichkeit sprachen ». (Die Ästhetik des Widerstands, op. cit., p. 82).

33. Celui-ci confortera Peter Weiss dans son intention de se démarquer de certaines visions du texte de Dante: «Zu dieser Zeit sah ich Gepeinigte vor ihren Peinigern stehn, letzte / Überlebende vor denen, die sie zur Tötung bestimmt hatten, und nicht mehr / Giganten waren es, nicht mehr Geister großer Dimensionen, nicht mehr / gefürchtete Priester, Stadtherren und Heerführer, und ihnen gegenüber / nicht mehr Heilige, Fromme, Gerechte, sondern nur / Namenlose auf beiden Seiten, nur Übriggebliebene / aus einer umfassenden Entwertung, nur Stammelnde, Verständnislose, / vor einem Gerichtshof, der trübe zerfließende Grausamkeiten ermittelte, / Grausamkeiten einförmig, tausendfach wiederkehrend, verschüttet, / ohne Farbe...» (Vorübung, op. cit, pp. 133-134).

34. Inferno se divise en 33 Chants, comme chacun des 3 volets de la Divine Comédie (en réalité 34 pour l'Enfer). Les chants de Inferno ont le statut de véritables scènes alors que les 11 Chants de Die Ermittlung seront des mini-actes subdivisés en trois parties.

35. «Peter Weiss im Gespräch mit A. Alvarez » (1964), in: Rainer Gerlach, Mathias Richter: Peter Weiss im Gespräch, Frankfurt am Main, Suhrkamp, 1986, p. 55.

Dans les quelques lignes de la version 9 d'Inferno, qui prépare directement le propos de Die Ermittlung, Dante-Aischmann souligne l'inadéquation de la conception dantesque de l'enfer en insistant sur l'impunité des anciens bourreaux et la nécessité de leur demander des comptes: «Damit können wir nichts mehr anfangen. Wir sehen die, die Schaden zufügen, hier zwischen uns, und wir sehen, es geht ihnen im allgemeinen gut. Sie nützen ihre Macht aus, sie bereichern sich nach bestem Vermögen, meistens werden sie bewundert und beneidet [...]. Können wir nicht ermitteln, wo Lügen ausgesprochen werden, wo Brutalitäten begangen werden, wo gestohlen wird, wo gemordet wird? Aber - du hast recht - die Sünder haben gute Verteidiger. » (Mü, p. 441).

36. Peter Weiss: Gespräch über Dante, op. cit., p. 168.

37. L'entretien, dont sont extraites les citations suivantes, a été reproduit in : Peter Weiss-Jahrbuch Bd. 4, Opladen, Westdeutscher verlag 1995, pp. 8-30.

38. La possibilité d'une extension de la problématique du "retournement de la morale de Dante » sera mise en doute au début de 1966, ce qui explique que Weiss cessa de travailler à la trilogie : on ne peut plus séparer le volet Paradiso de celui de Inferno, le monde des bourreaux et celui des victimes qui doivent «être en permanence confrontés l'un à l'autre » écrit Weiss à Unseld le 1.3.1966 (Cité in : Mü, p. 239).

39. Dans la version achevée d'Inferno, le chef devenu «Phlegias», repris du 5e cercle dantesque des «colériques", tient au Chant 13 le discours canonique de l'exclusion exterminatrice du national-socialisme ("Parasiten », "Schmarotzer », "Giftgeschwür», "Seuche ») - qu'aurait écouté sans protester le Dante d'avant l'exil (Chant 12) - avant de déclamer au Chant 19 la tirade du pouvoir qui avait su renaître des cendres de ses propres destructions : «Grösser als je / ist heute unsre Macht /.../ Erst durch die Zerstörung / sind wir zu diesem Überfluss gekommen ». Louange des vivants qui ont survécu à l'effondrement et prônent sans vergogne l'oubli des corps "détruits par la pourriture » ou "partis en fumée » : "Wer fragt nach denen / die verwesten und verrauchten / Nur mit den Lebenden wird hier gerechnet ». On peut citer aussi la longue 
réplique de Dante au début du Chant 19 d'Inferno (p. 65) : reconnaissant sa culpabilité passée qui fut adéquation et allégeance à l'image que les bourreaux attendaient de lui, celle d'un faible saisi par une furie barbare à l'égard des autres faibles, il déclare: «In meiner Schwäche schaffte ich mir / Gift und Krallen / um so zu sein / wie ihr mich sehen wolltet / Ich lernte es von Anfang an / es wurde aus mir herausgeschlagen / Mich schlug die Furie / und ich schlug weiter /.../ ich war bereit / dem Schwachen ins Gesicht zu schlagen ». La tirade se termine par « und doch gehörte ich nie / zu euch » qui sonne en partie comme une dénégation.

40. Citations in: Inferno, op. cit., pp. 13-14.

41. Dans la version 8.2, la formule dantesque est conservée et explicitée par Charon: "Das Aufgeben jeglicher Hoffnung ist gleichbedeutend / mit dem Aufgeben der Illusionen / Keine Veränderung wird von aussen erwartet /.../ Die Vorstellungen von Gut und Böse von Schönheit oder Hässlichkeit / werden aufgehoben im Verzicht auf alle Hoffnung » (Mü, p. 437).

42. Ce consentement masochiste est d'ailleurs mis en scène au Chant 21 de la version achevée: « Ach wir sind arm doch sind wir glücklich » - « Wir danken euch / dass ihr uns tretet » (p. 74).

43. Cf. le début de la Vorübung où Weiss explique s'être dans un premier temps davantage intéressé à Giotto le réaliste, peintre de l'ici-bas, qu'à Dante le visionnaire du destin des humains dans l'au-delà. On peut citer un bref passage des Notizbücher 1960-71 qui date du tout début du travail sur Inferno : « Dante und Giotto wandern durch die Konzentrationslager. / Frage: lässt sich dies noch beschreiben. / Szene des völligen Schweigens. / Der tiefsten Trauer. / Können wir weiterleben nach diesem. » (op. cit., p. 215).

44. Dans la version achevée d'Inferno, le peintre est au Chant 26 l'un des masques de Virgile qui reprend l'opposition Dante/Giotto de la Vorübung en reprochant à Dante son inaccessibilité d'habitant de la tour d'ivoire réfugié dans «l'atmosphère glacée de la perfection formelle » aux antipodes du «monde des corps» qui était le sien, avec ses «bosses, ses obscurités et ses dangers » (p. 93).

45. Il y est encore un compagnon de cheminement qui partage la vision d'un Enfer/Paradis de tous les bourreaux : "Ich werde dich leiten durch ihr Reich das besteht das noch dasteht fest doch nicht ewig das Reich in dem sie verbannt sind: die Mörder die Schänder die Betrüger die Räuber die Fälscher die Verführer » (Mü, p. 335).

46. Le « Aischmann-Entwurf » met en scène un Virgile médecin beaucoup plus introspectif, qui se dit mort vivant («Ich stehe auf der Seite der Toten »), mort au monde et à sa propre conscience pour avoir accepté de servir le pouvoir des assassins qui ont mis la société en coupe réglée (Mü, p. 423).

47. Au chant 4, Charon, reprit de la Divine Comédie et transformé en hypostase du «Chef/Patron », donnera pour consigne à Dante de ne pas chercher à donner d'un réel éternellement renouvelé une représentation qui excède les apparences qu'il se donne : "Hier brauchst du nicht nach Gleichnissen zu suchen / Was sich dir zeigt / das ist sich selbst genug / Sieh wie hier alles lebt und sich bewegt / und wie hier nichts verharrt / im Alten und Verbrauchten / Bei uns ist jeder Augenblick / Erneuerung. » (Inferno, p. 22)

48. Il le demeure néanmoins, comme le montre le Chant 6 de la Dante-Prosa : même s'il se pose plus en nom-signifiant qu'en figure historico-littéraire - «...nenne mich Vergil, mir ist es recht, wenn dir nichts anderes einfällt »-sa fonction de séide du pouvoir, chargé de «transfigurer les diverses activités rassemblées sous la notion de culture » et de transformer Dante en monument culturel, n'est que temporairement remise en question (Mü, 520).

49. Ainsi dans les versions 2, 3.1 et 3.4 publiés dans les annexes du livre de Müllender.

50. Dans Homo sacer Agamben décrit le retour contemporain de cet exclu du corps social de la communauté juridico-politique de l'Empire romain et réduit à une existence nue, sans droits ni protections. C'est une sorte de « mort vivant " pour qui l'état d'exception est la règle, le « camp » des régimes totalitaire représentant le « paradigme biopolitique de la modernité ». 
51. Peut-on voir là une allusion, d'une forte consistance intertextuelle, à un autre «Gegenwort » célèbre de la littérature allemande, le : «Ich sage mich vom Gesetz der Frauen für immer los!» de la Penthésilée de Kleist? Il n'est pas inintéressant de noter que, dans l'opéra que Johannes Kalitzke a tiré d' Inferno en 2006, cette ultime réplique est mise dans la bouche de Béatrice qui y fonctionne comme une sorte de spectre alors qu'elle n'a dans la pièce qu'une présence narrée. Cf. Claudia Heinrich: "Zur Kenntlichkeit ent-stellt. Vergleichende Analyse des Librettos von Johannes Kalitzke zur Operninszenierung Inferno und des Textes des gleichnamigen Theaterstücks von Peter Weiss » in: Peter Weiss-Jahrbuch Bd. 15, Röhrig Universitäts-Verlag, St. Ingbert 2006, pp. 69-96.

52. «'Es ist eine Wunschautobiographie'. Peter Weiss im Gespräch mit Rolf Michaelis über seinen politischen Gleichnisroman », cité par Y. Müllender, op. cit., p. 276, n. 7.

53. Une note des Notizbücher sur les dissensions lors de la rencontre de Princeton en avril 1966 Weiss avait critiqué publiquement la guerre américaine au Vietnam - est particulièrement explicite : "Auch Kritik an Deutschland, sagte ich, hielte ich nicht zurück, weil ich in Schweden ansässig sei. Und dann kam es: du kannst dich über Deutschland nie äußern, du bist draußen gewesen, in der Sicherheit der Emigration, wir waren drinnen, wir haben am Krieg teilgenommen - Dies war, was ich immer wieder gespürt hatte, wenn ich in Deutschland war, und was oft im Ungewissen blieb: dieser einmal vollzogne, definitiv gewordne Bruch - » (Notizbücher 1971-1980, S. 734). Il n'est pas impossible que le Groupe 47 soit implicitement critiqué dans Inferno comme une frange ambivalente de la «Kulturindustrie » des années cinquante et soixante en RFA. Weiss notera dans ses Notizbücher 1971-1980 son sentiment d'avoir été d'emblée perçu et reçu comme un élément extérieur et étranger à qui on pouvait reprocher plus ou moins explicitement son émigration. La représentation de la «Heimkehr» du Dante de Inferno est certainement nourrie de ces biographèmes.

Voir là-dessus l'article d'Helmut Müssener: «'Du bist draußen gewesen'. Bemerkungen zur unmöglichen Heimkehr des Schriftstellers Peter Weiss » in: J. Fetscher u.a.: Die Gruppe 47 in der Geschichte der Bundesrepublik, Würzburg 1991.

Th. Mann pointait dès 1954 ce qu'il ressentait comme une corruption arrogante de la littérature de «ladite jeune génération » par le renouveau économique dans une Allemagne américanisée : «Ich kenne die Unverschämtheit der sogenannten jungen Generation [...]. Sie hängt wohl auch mit der lächerlichen Wirtschaftsblüte der amerikanischen Lieblingskolonie ,Westdeutschland' zusammen, diesem frechen und unmoralischen Wohlsein nach Schandtaten, die mit der Höllenfahrt von 1945 schlossen und an die heute zu erinnern nichts weiter als bolschewistisch ist. » (Lettre du 17 mai 1954 à Klaus Mampell, cité in: Heinz Ludwig Arnold, Die Gruppe 1947, Rowohlt, Reinbek bei Hamburg 2004, p. 149).

54. «Unsre Krankheiten sind zumeist politische Krankheiten. Wenn uns der Atem wegbleibt, wenn das Blut in den Adern stockt, das Herz aussetzt, dann hat sich unser Überdruß im Organismus eingenistet, dann reagieren wir mit unsrer ganzen Person, als Einheit, als Naturprozeß, definitiv, auf eine Situation, der mit der Vernunft nicht mehr beizukommen ist» (P. Weiss: Die Notizbücher, Digitale Bibliothek, S. 11610).

55. «... unter uns verweste Gebeine Asche verfilztes Haar / ausgeschlagene Zähne gespaltene Schädel / .../ ein irrsinniges Tier ist der Mensch» (P. Weiss: Die Verfolgung und Ermordung JeanPaul Marats, Suhrkamp, Frankfurt am Main, 1965, p. 46).

56. Pendant la première année londonienne, en 1935, il expose ses premières toiles dont il dira dans Abschied von den Eltern: "Ich schilderte [...] meine Visionen von apokalyptischen Landschaften mit Feuersbrünsten, fliehenden Tieren, Ertrinkenden und vergehenden Städten, meine Visionen von Gekreuzigten und Gegeißelten, von schrecklichen Fratzen und verführerischen Frauengesichtern. »- « Mes visions de paysages apocalyptiques, incendies, bêtes en panique, hommes noyés, villes détruites, visions de crucifixion et de flagellation, d'horreurs grimaçantes et de séduction féminine.» Entre Dante, le «Höllen-Breughel» et Hieronymus 
Bosch. Si l'on en croit Abschied, la plupart seront détruites à coup de haches puis brûlées par la mère de Weiss : « Mit ihren eigenen Händen hatte sie die Bilderwelt meiner Jugendjahre, diese Totentänze, Weltuntergänge und Traumlandschaften vernichtet. Mit dieser Vernichtung hatte sie sich von der Drohung befreit, die diese Bilder auf die Geordnetheit und Behütetheit ihres Heims ausgeübt hatten. » (P. Weiss: Abschied von den Eltern, Suhrkamp, Frankfurt am Main, 1964, pp. 108 et 138).

On peut penser que la réécriture de l'Enfer de Dante est aussi un retour sur une certaine production picturale qui, dans l'après coup, générera des significations nouvelles en s'adossant aux récits des témoins de la Shoah.

57. Citations in : Die Ästhetik des Widerstands, op. cit., p. 83.

58. La thématique de la non-résistance des opprimés à l'oppression sera développée à mainte reprise dans Die Ästhetik des Widerstands.

59. «Ich Phlegias spreche zu euch von den Parasiten / die gekommen sind in unsre Stadt / zuerst sich krümmend vor uns und uns dienend / bald aber an sich raffend in unverstellter Selbstsucht... » (Inferno, p. 48).

60. La citation est extraite de l'auto-portrait qui orne à juste titre la quatrième de couverture du magistral Peter Weiss Leben und Werk publié par G.Palmstierna-Weiss et Jürgen Schutte en bilingue (allemand/suédois) (Suhrkamp, Frankfurt am Main 1991): "Dieses In-GegensätzenDenken - angefressen von der Zweifel-Krankheit, Schwierigkeit, mich für eine Sache zu entscheiden, Hin und Her, Schwanken in der Arbeit, früher Malen - Schreiben, Theater -Film schwedische - deutsche Sprache, reisen hierhin, dorthin, dieses ständige Auspeilen, es ist, als sei mein Wesen zusammengesetzt aus den beiden im Streit liegenden Polen, zwischen diesen wird alles ausgemacht, immer wird Gegensätzliches von mir verlangt, das ist meine Triebkraft, die all meine Arbeit erzeugt ».

61. Müllender cite une interview de Gunilla Palmstierna-Weiss d'août 1986 selon laquelle Peter Weiss avait l'intention, peu de temps avant sa mort, de se consacrer au Purgatorio (Mü, 295).

62. Vorübung, op. cit., p. 137.

63. Dans le Notizbuch 17, le „Purgatorio“ représente de plus en plus un «appendice du Paradiso »: "zum Schluss: es gibt nichts als Hass, unversöhnlichen Hass, Brandmarken, Rache, äußerste Gewalt, unermüdliche Anstrengung, den Feind zu vernichten » (Peter Weiss: Die Notizbücher,op. cit,p. 11546).

64. Cette dénomination est répétée dans la Dante-Prosa et pourrait dénoter une référence (inversée ?) à ce par quoi Brecht désignait le socialisme avant de parler plutôt de "grande production » dans une note de Journal du 7.3.41.

\section{RÉSUMÉS}

Inferno de Peter Weiss fut le premier volet d'une trilogie théâtrale inachevée, tramée sur la Divine Comédie de Dante. La pièce fut écrite fin 1964 et publiée pour la première fois en 2003 tandis que le troisième volet Paradiso parut en 1965 avant d'être représenté simultanément pour la première fois sur plusieurs scènes ouest- et est-allemandes sous le titre Die Ermittlung. De cette première partie du « Projet Divine Comédie », il existe plusieurs versions et un assez long texte en prose de 1969 qui marque un tournant significatif dans ce projet et en est une sorte de clôture. "Selon Dante, l'enfer est le lieu où les pécheurs subissent des tourments infernaux éternels. Mais, de nos 
jours, ils ne sont pas punis, ils ne sont pas en enfer. (...) Ces hommes qui auraient dû être punis ont une existence très confortable: ils exercent leur domination et détiennent le pouvoir. Et ceux qui devraient être au paradis vivent dans la misère éternelle qu'ils ont subie ", écrivait Weiss. Il s'agissait donc d'opérer un retournement de l'architecture de l'œuvre de Dante et la transformation du je-narrateur d'un vaste poème épique ( Weltgedicht») en personnage dramatique distancié d'un " grand théâtre du monde ». Au lieu des événements mettant en scène une histoire italienne à la fois réelle et imaginaire adossée à de "grands récits » - mythologie antique ou religion chrétienne - c'est l'histoire de l'Allemagne d'après-guerre et de son passé le plus récent qui devait être mise en scène avec un autre travail de réécriture après coup d'éléments autobiographiques essentiels : complexe de culpabilité du « survivant ", rapport à une émigration qui ne fut pas exil mais préserva le "demi-juif» Peter Weiss d'une probable extermination, rapport critique à la «restauration» socio-économique et idéologique de l'Allemagne d'Adenauer dans laquelle le citoyen suédois Peter Weiss séjourna régulièrement dans les années soixante et qui fut la scène ambivalente de ses succès et de ses déceptions, définition critique du statut de l'écrivain «dans un monde divisé » où l'engagement politique était à la fois une nécessité et peut-être aussi une impossibilité.

Peter Weiss' Inferno bildete den ersten Teil einer unvollendeten Theatertrilogie, die im Rahmen eines « Projektes Divina Commedia » entstand. Der Ende 1964 fertig gestellte Inferno-Teil, von dem es mehrere Fassungen mit einem längeren Prosatext gibt, erschien erst posthum im Jahre 2003, während der Paradiso-Teil unter dem Titel Die Ermittlung 1965 veröffentlicht und auf mehreren west- und ostdeutschen Bühnen gleichzeitig uraufgeführt wurde. „Im Inferno werden nach Dantes Vorstellung die Sünder mit ewigen Höllenqualen bestraft. Aber heute werden sie nicht bestraft und sind nicht in der Hölle. (...) Diese Menschen, die an sich bestraft werden müssten, leben sehr bequem unter uns als Herrschende und Machthaber. Und die im Paradies leben sollten, leben ewig in dem Elend, das sie durchgemacht haben“, schrieb Weiss. Es galt also zunächst, die Gesamtarchitektur des Danteschen „Weltgedichts“ umzukrempeln und den IchErzähler in die verfremdete dramatische Hauptperson eines „Welttheaters“ zu verwandeln. Dadurch sollte nicht mehr eine zugleich reale und imaginäre, von den „großen Erzählungen“ der antiken Mythologie oder der christlichen Religion gestützte, italienische Geschichte, sondern die Geschichte Nachkriegsdeutschlands und dessen jüngster Vergangenheit inszeniert werden. Dabei wurden wesentliche autobiographische Elemente hinterfragt und neu bewertet: Überlebensschuld, subjektive Einordnung der nicht zum Exil stilisierbaren Emigration, die jedoch den „Halbjuden“ Peter Weiss vor einer wahrscheinlichen Vernichtung bewahrte, Beziehung zum Westdeutschland der Adenauerschen Restauration, in dem er sich in den sechziger Jahren mehrmals aufhielt und die zum ambivalenten Schauplatz seiner Erfolge und seiner Enttäuschungen wurde, kritische Definition des Standorts des Schriftstellers in einer „geteilten Welt", in der das politische Engagement zugleich eine Notwendigkeit und eine Unmöglichkeit war.

INDEX

oeuvrecitee Inferno, Dante-Prosa

\section{AUTEURS}

ANDRÉ COMBES

Université Toulouse Le Mirail 